This is a post-peer-review, pre-copyedit version of an article published in Operational Research. The final authenticated version is available online at:

https://doi.org/10.1007/s12351-019-00534-9 


\title{
New Concepts and an Algorithm for Multiobjective Bilevel Programming: Optimistic, Pessimistic and Moderate Solutions
}

\author{
Maria João Alves ${ }^{1,3}$ [0000-0002-2268-0110] *, Carlos Henggeler Antunes ${ }^{2,3}$ [0000-0003-4754-2168], \\ João Paulo Costa ${ }^{1,3}$ [0000-0001-5204-3916] \\ ${ }^{1}$ CeBER and Faculty of Economics, University of Coimbra, Portugal \\ ${ }^{2}$ DEEC, University of Coimbra, Polo 2, Coimbra, Portugal \\ ${ }^{3}$ INESC Coimbra, Portugal \\ mjalves@fe.uc.pt; ch@deec.uc.pt; jpaulo@fe.uc.pt
}

\begin{abstract}
Bilevel optimization deals with hierarchical mathematical programming problems in which two decision makers, the leader and the follower, control different sets of variables and have their own objective functions subject to interdependent constraints. Whenever multiple objective functions exist at the lower-level problem, the leader should cope with the uncertainty pertaining to the follower's reaction. The leader can adopt a more optimistic or more pessimistic stance regarding the follower's choice within his efficient region, which is restricted by the leader's choice. Moreover, the leader may also have multiple objective functions. This paper presents new concepts associated with solutions to problems with multiple objective functions at the lowerlevel and a single or multiple objective functions at the upper-level, exploring the optimistic and pessimistic leader's perspectives and their interplay with the follower's choices. Extreme solutions (called optimistic/deceiving and pessimistic/rewarding) and a moderate solution, resulting from the risk the leader is willing to accept, are defined for problems with a single objective at the upper-level (semivectorial problems). Definitions of optimistic and pessimistic Pareto fronts are proposed for problems with multiple objective functions at the upper-level. These novel concepts are illustrated emphasizing the difficulties associated with the computation of those solutions. In addition, a differential evolution algorithm, approximating the extreme and moderate solutions for the semivectorial problem, is presented. Illustrative results of this algorithm further stress the challenges and pitfalls associated with the computation and interpretation of results in this kind of problems, which have not been properly addressed in literature and may lead to misleading conclusions.
\end{abstract}

Keywords: multiobjective bilevel optimization; semivectorial bilevel problem; optimistic and pessimistic Pareto fronts; optimistic, deceiving, pessimistic, rewarding and moderate solutions.

Mathematics Subject Classification: 90B50, 90C26, 90C29

\footnotetext{
${ }^{*}$ Corresponding author. Tel. +351239790558
} 


\section{Introduction}

Bilevel optimization deals with hierarchical non-cooperative decision processes in which the upper-level decision maker (the leader) and the lower-level decision maker (the follower) control different sets of variables and have their own objective functions. That is, the lower-level problem is embedded in the constraints of the upper-level problem. The leader sets first the values of his variables $x$ to optimize his objective function, which establishes the feasible region for the follower's problem. The follower then determines the values of his variables $y$ that optimize his objective function within this feasible region. Hence, the leader needs to consider the follower's reaction to the setting of $x$ since this influences feasibility and the leader's objective value. Bilevel optimization models are useful in problems involving hierarchical decision processes such as resource management, e.g. relating to planning and operational decision levels, and policymaking, e.g. regarding the definition of pricing policies (Labbé and Violin, 2013). Overviews of bilevel optimization with surveys of algorithms and applications can be found in (Vicente and Calamai, 1994), (Bard, 1998), (Dempe, 2002), (Colson, Marcotte and Savard, 2005), (Colson, Marcotte and Savard, 2007), and (Sinha, Malo and Deb, 2018).

The multiobjective bilevel problem (MOBP) encompasses multiple objective functions at one or both levels. This paper deals with the MOBP in which the lower-level problem has multiple objective functions, whereas the upper-level problem may have a single or multiple objective functions. The first case is generally designated as semivectorial bilevel problem (SVBP). If multiple objective functions exist at the lower-level, then a set of lower-level efficient solutions exists for each leader's setting of $x$ variables. Therefore, the leader should foresee the follower's choice within his efficient solution set for any instantiation of the $x$ variables. Approaches assuming that the leader has information about the follower's preferences, thus enabling to know the follower's choice within his efficient region, would facilitate the analysis. Indeed, if the leader knew the follower's utility function aggregating his multiple objectives, the uncertainty about the lower-level decision would be eliminated. However, this is not realistic in practice and the lower-level efficient region should be explicitly considered to find the optimal or efficient solutions to the upper-level problem.

Almost all works on SVBP and MOBP described in literature have adopted an optimistic approach, i.e., they assume that the follower accepts any efficient decision to the lower-level problem or, equivalently, chooses among his efficient decisions according to the leader's preferences (Ruuska and Miettinen, 2012). The optimistic approach is also the most often considered in single-objective bilevel optimization when alternative optima exist for the follower's problem, since the pessimistic bilevel formulation is perceived to be more difficult to solve. However, the nature of the decisions taken by the follower in SVBP/MOBP is quite different from the decisions in single objective bilevel optimization. In the single-objective case, the follower's reaction solutions are alternative optima with the same value for a single objective function. In SVBP/MOBP, there is a set of nondominated (efficient) decisions representing different tradeoffs among the objectives and one of them is chosen by the follower. It is hardly realistic to consider that the follower is indifferent to all these solutions that are possible for a given leader's decision. Consider, for instance, a tollsetting problem. The owner of a highway system (leader) sets tolls and wants to maximize total revenue. The users of highways (follower) want to minimize travel costs and minimize travel times. For a given leader's decision - toll prices - there is a set of nondominated decisions to the users, ranging from the minimum cost solution to the minimum time solution. The minimum time solution (maximum cost) is the one that provides the leader the maximum revenue. The optimistic approach would lead the leader to set high toll prices under the (very unlikely) belief that users would use highways regardless the price.

Therefore, the leader may adopt different attitudes regarding his expectation of the follower's choice. Those leader's attitudes may be more optimistic or more pessimistic vis-à-vis his anticipation of the follower's decision being more or less favorable. The combination of different leader's attitudes and follower's responses results in different types of solutions that should be exploited to provide useful information about possible outcomes and aid the leader in his decision process. For this purpose, novel concepts are proposed to the SVBP and the MOBP: in addition to the optimistic and pessimistic solutions, definitions of the deceiving, rewarding and moderate solutions are stated for the SVBP and the optimistic and the pessimistic Pareto fronts for the MOBP. All these concepts are thoroughly explained herein using illustrative graphical examples, which help to emphasize the difficulties associated with the calculation (or approximation) of these solutions.

The works reported in the scientific literature devoted to SVBP have mainly addressed the optimistic formulation, i.e. assuming that the follower always chooses the solution of his efficient set that is the best for the leader. The SVBP was first dealt with by Bonnel (Bonnel, 2006), who developed necessary optimality conditions for an optimistic formulation. Ankhili and Mansouri (Ankhili and Mansouri, 2009), Zheng and Wan (Zheng and Wan, 2011), Zheng et al. (Zheng, Chen and Cao, 2014), and Ren and Wang (Ren and Wang, 
2016) proposed penalty function methods to compute the optimistic solution to the SVBP with a multiobjective linear programming (MOLP) problem at the lower-level. Calvete and Galé (Calvete and Galé, 2011) tackled the SVBP with a MOLP at the lower-level problem reformulating it as an optimization problem over a nonconvex region guaranteeing that the optimistic solution is an extreme point of the feasible polyhedron. Lv and Wan (Lv and Wan, 2014) used the weighted-sum scalarization to reformulate the linear SVBP into a single objective bilevel problem where the lower-level is a parametric linear scalar problem, which is then converted into a single level nonlinear, nonconvex programming problem with a linear objective function.

The pessimistic formulation of the SVBP assumes that the follower always chooses the solution of his efficient set that is the worst for the leader. Liu et al. (Liu et al., 2014) converted the pessimistic SVBP into a generalized minimax optimization problem with constraints and developed necessary optimality conditions for the pessimistic formulation. Lv and Chen ( $\mathrm{Lv}$ and Chen, 2016) computed the pessimistic solution to a SVBP with a convex lower-level problem without upper-level variables in the constraints. Alves et al. (Alves, Antunes and Carrasqueira, 2015) introduced the concept of deceiving solution and proposed a Particle Swarm Optimization (PSO) algorithm to compute the optimistic, pessimistic and deceiving solutions to the SVBP. (Alves and Antunes, 2018a) then presented the concept of rewarding solution and developed a differential evolution algorithm to compute the four extreme solutions. These four extreme solutions are also illustrated in (Alves, Antunes and Costa, 2019) and their interest in real-world applications has been exploited in a case study dealing with the optimization of dynamic time-of-use electricity retail prices (Alves and Antunes, 2018b). These solutions will be presented in detail in sections 2 and 3.

The MOBP was addressed by Shi and Xia (Shi and Xia, 1997) transforming it into separate multiobjective problems at each level, using in addition a satisfactoriness concept to model the leader's preferences. The interactive incorporation of the leader's preferences to compute satisfactory solutions was also used by (Shi and Xia, 2001) and (Abo-Sinna and Baky, 2007). New theoretical results for the nonlinear nonconvex MOBP were proposed by Eichfelder (Eichfelder, 2010). For the linear MOBP, Pieume et al. (Pieume et al., 2011) proposed the use of a surrogate single level MOLP problem to generate the complete set of feasible solutions. The leader's preferences are then aggregated using a weighted-sum of the upper-level objective functions. However, this scheme is difficult to implement because it consists of optimizing an objective function over the efficient set of a MOLP problem. All these works consider an optimistic formulation of the MOBP problem, assuming that the leader can choose the follower's efficient solutions most convenient for himself. The concept of optimistic Pareto front in MOBP is further illustrated in (Alves, Antunes and Costa, 2019).

Metaheuristic approaches have been developed to approximate the Pareto front of the MOBP in order to circumvent the difficulties of its exact computation. These approaches have also considered an optimistic formulation: Deb and Sinha (Deb and Sinha, 2010) proposed a hybrid evolutionary algorithm with a local search phase; Zhang et al. (Zhang et al., 2013) developed a hybrid PSO algorithm with a crossover operator using an elitist strategy; Carrasqueira et al. (Carrasqueira, Alves and Antunes, 2015) used a multiobjective PSO algorithm, paying special attention to the need to ensure that lower-level solutions are truly efficient to be feasible to the MOBP.

Gupta and Ong (Gupta and Ong, 2015) converted the lower-level problem into a single-objective problem by means of scalarization techniques with adaptive parameters (e.g. weights). This process configures an optimistic approach, since these weights are then included as leader's decision variables, thus meaning that the leader can determine the most advantageous weights for the follower's objectives. Ruuska and Miettinen (Ruuska and Miettinen, 2012) developed a procedure to construct evolutionary algorithms for the optimistic MOBP.

Sinha et al. (Sinha et al., 2016) considered that the follower's preferences are represented by a value function, which is parameterized by an uncertain preference vector, and proposed a two-step approach: the Pareto front is obtained for fixed parameters using the leader's anticipation about the follower's preferences and then the leader estimates a confidence region around that Pareto front. Sinha et al. (Sinha, Malo and Deb, 2017) classify the MOBP into: the optimistic formulation; deterministic decisions at lower-level considering the leader has perfect information about the follower's preference structure; lower-level decision uncertainty considering that the follower's preferences are modeled by a random variable.

This paper proposes novel solution concepts in SVBP and MOBP, using illustrative graphical examples to shed light on the insights these solutions can offer to support decision processes. The existence of a lowerlevel efficient region requires the adoption by the leader of a more optimistic or a more pessimistic attitude, thus preparing for the follower's reaction more in favor or more adverse to the leader's objectives. This interplay between the leader's and the follower's decisions are explored to define: in SVBP, the optimistic and the pessimistic frontiers, where the extreme optimistic/deceiving and pessimistic/rewarding solutions are 
located, as well as a moderate solution resulting from the risk the leader is willing to accept vis-à-vis the follower's choice; in MOBP, the optimistic and the pessimistic Pareto fronts.

In face of the difficulties to determine exact solutions, we present an algorithm based on differential evolution (DE), which extends the algorithm proposed in (Alves and Antunes, 2018a) to compute the four extreme solutions plus moderate solutions to the SVBP. The analysis of the illustrative results obtained with this DE approach enables to unveil pitfalls associated with the interpretation of results and assessment of algorithms in SVBP and MOBP. These pitfalls have not been properly addressed in the literature, which may lead to misleading conclusions.

Therefore, the main aim of this paper is to offer solution concepts and focus on relevant issues from the decision aid point of view, rather than establishing mathematical conditions for the existence of these solutions or necessary conditions for optimality. The moderate solution to the SVBP and the pessimistic Pareto front to the MOBP with multiple objective functions at both levels are for the first time presented in this paper. The problems addressed are difficult and, even when those solutions do exist, it can be impracticable to compute them exactly. Therefore, the use of heuristics and metaheuristics becomes imperative in most cases to compute (expectedly good) approximate solutions.

The paper is organized as follows. In section 2, the MOBP is formulated and the notation is introduced. The SVBP is presented in section 3, illustrating the concepts of optimistic/deceiving and pessimistic/rewarding solutions located on the optimistic and pessimistic frontiers. Section 4 deals with the leader's decision risk in SVBP, proposing the concept of moderate solution. The bilevel problem with multiple objective functions at both levels is addressed in section 5, for which definitions of optimistic and the pessimistic Pareto fronts are proposed and illustrated using linear and nonlinear examples with specific features. Section 6 presents a DE algorithm to approximate the extreme and moderate solutions to the SVBP and discusses illustrative results. Conclusions are drawn in section 7 , stressing some pitfalls associated with the computation of solutions to the SVBP/MOBP.

\section{$2 \quad$ Multiobjective Bilevel Programming}

Let us consider the multiobjective bilevel programming (MOBP) problem with $k \geq 2$ objective functions at the upper-level (leader), or $k=1$ as a particular case, and $m \geq 2$ objective functions at the lower-level (follower). This problem can be formulated as follows:

$$
\begin{array}{ll}
\max _{x} & \left(F_{1}(x, y), \ldots, F_{k}(x, y)\right) \\
\text { s.t. } & G(x) \leq 0 \\
& y \in \underset{y}{\arg \max _{y}}\left\{\left(f_{1}(x, y), \ldots, f_{m}(x, y)\right): g(x, y) \leq 0\right\}
\end{array}
$$

where $x \in \mathbb{R}^{n_{1}}$ are the decision variables controlled by the leader and $y \in \mathbb{R}^{n_{2}}$ are the decision variables controlled by the follower. In this formulation, we are assuming that the upper-level constraints $G(x) \leq 0$ only depend on the upper-level decision variables $x$, thus not involving the lower-level decision variables $y$. $g(x, y) \leq 0$ are general constraints, involving both $x$ and $y$, in the lower-level problem.

If a single objective function exists at the upper-level $(k=1)$ and $m \geq 2$, the problem is generally designated as semivectorial bilevel problem (SVBP).

$$
\begin{array}{ll}
\max _{x} \quad & F(x, y) \\
\text { s.t. } & G(x) \leq 0 \\
& y \in \underset{y}{\arg \max _{y}}\left\{\left(f_{1}(x, y), \ldots, f_{m}(x, y)\right): g(x, y) \leq 0\right\}
\end{array}
$$

Since the leader has control only over $x$, the quotation marks in "max" aim to denote the imprecise definition of the objective function value(s) $F(x, y)\left(F_{k}(x, y)\right)$ from the leader's perspective because, in general, a (not singleton) set of efficient solutions to the lower-level problem exists.

For each upper-level decision $x$, only efficient (Pareto optimal) solutions to the lower-level problem are feasible to the MOBP/SVBP. Let $X=\left\{x \in \mathbb{R}^{n_{1}}: G(x) \leq 0\right\}, Y(x)=\left\{y \in \mathbb{R}^{n_{2}}: g(x, y) \leq 0\right\}$ and assume that $Y(x) \neq \varnothing$ for $\forall x \in X$. 
Definition 1 - $f$-efficient solution

For a given $x^{\prime} \in X$, a solution $y^{\prime} \in Y\left(x^{\prime}\right)$ is efficient to the lower-level problem (f-efficient) if and only if there is no other $y \in Y\left(x^{\prime}\right)$ that dominates $y^{\prime}$, i.e. such that $f_{j}\left(x^{\prime}, y\right) \geq f_{j}\left(x^{\prime}, y^{\prime}\right)$ for all $j=1, \ldots, m$, and $f_{j}\left(x^{\prime}, y\right)>f_{j}\left(x^{\prime}, y^{\prime}\right)$ for at least one $j$.

Therefore, the set of all $f$-efficient solutions for a given $x^{\prime} \in X$ can be defined as:

$\Psi_{\mathrm{Ef}}\left(x^{\prime}\right)=\left\{y^{\prime} \in Y\left(x^{\prime}\right)\right.$ : there is no $y \in Y\left(x^{\prime}\right)$ such that $\left.\boldsymbol{f}\left(x^{\prime}, y\right) \succ \boldsymbol{f}\left(x^{\prime}, y^{\prime}\right)\right\}$ where $\succ$ denotes the dominance relation and $\boldsymbol{f}(x, y)$ is the objective vector $\left(f_{1}(x, y), \ldots, f_{m}(x, y)\right)$.

The induced region (i.e, feasible region) of the MOBP/SVBP is:

$$
I R=\left\{(x, y) \in \mathbb{R}^{n_{1}} \times \mathbb{R}^{n_{2}}: x \in X, y \in \Psi_{\mathrm{Ef}}(x)\right\} .
$$

For each leader's decision $x^{\prime}$, the follower has, in general, a set of efficient solutions. Therefore, the leader should prepare for any compromise solution selected by the follower within that efficient solution set, which may be more or less favorable according to the leader's single objective (SVBP) or multiple objectives (MOBP). This lack of knowledge about the follower's preferences leading to the choice of a particular efficient solution compels the leader to adopt an attitude in face of his expectation of the follower's choice. In fact, it is seldom realistic to consider that the follower is indifferent to all resulting efficient solutions for a given leader's decision $x^{\prime}$, or he chooses according to the leader's objectives.

The more optimistic or more pessimistic attitude adopted by the leader foreseeing the follower's choice leads to the computation of different solutions, which provide decision support information regarding possible outcomes that define reachable ranges for the upper-level objective function values.

\section{Extreme solutions to the SVBP}

In the optimistic approach (optimistic formulation) to the SVBP, the leader chooses the $x$ assuming that the follower selects the solution within $\Psi_{\mathrm{Ef}}(x)$ that gives the best $F$ for the leader.

The optimistic formulation of the SVBP can be stated as follows, in which the upper-level optimization is taken with respect to $x$ and $y$ :

Definition 2 - Optimistic solution

$$
\begin{array}{ll}
\max _{x, y} & F(x, y) \\
\text { s.t. } & G(x) \leq 0 \\
& y \in \Psi_{\mathrm{Ef}}(x)
\end{array}
$$

An optimistic solution, $\left(x^{\mathrm{o}}, y^{\mathrm{o}}\right)$, is a solution that optimizes the optimistic formulation.

Thus, in the optimistic formulation of the SVBP, the solution to the lower-level problem for each $x$ is the $f$-efficient solution offering the best value of the upper-level objective function.

In the pessimistic approach (pessimistic formulation) to the SVBP, the leader chooses the $x$ that leads to a solution with best $F$ in face of the follower's decisions $y$ that are the worst for the leader.

The pessimistic formulation of the SVBP can be stated as follows, in which a max-min problem is considered at the upper-level:

$$
\begin{array}{ll}
\max _{x} \min _{y} F(x, y) & \\
\text { s.t. } & G(x) \leq 0 \\
& y \in \Psi_{\mathrm{Ef}}(x)
\end{array}
$$


Definition 3 - Pessimistic solution

A pessimistic solution, $\left(x^{\mathrm{p}}, y^{\mathrm{p}}\right)$, is a solution (if it exists) that optimizes the pessimistic formulation.

Thus, in the pessimistic formulation of the SVBP, the solution to the lower-level problem for each $x$ is the $f$-efficient solution leading to the worst value of the upper-level objective function.

From a theoretical perspective, the existence of the pessimistic solution requires more restrictive assumptions than the optimistic solution (see e.g. (Lucchetti, Mignanego and Pieri, 1987), (Dassanayaka, 2010)). Figure 1 illustrates a case where no pessimistic solution exists. Let $\varphi_{p}(x)=\min _{y}\left\{F(x, y): y \in \Psi_{\mathrm{Ef}}(x)\right\}$ be the worst upper-level objective function values achievable within the efficient reaction set for each $x$. The pessimistic solution is the one that maximizes $\varphi_{p}(x)$ for $x \in X$. The pessimistic solution does not exist but solutions very close to it exist, as $x_{0}^{\prime}$ in the figure. This solution can be considered a good approximation to the pessimistic solution, which can be useful for the leader's decision process in the context of a practical problem.

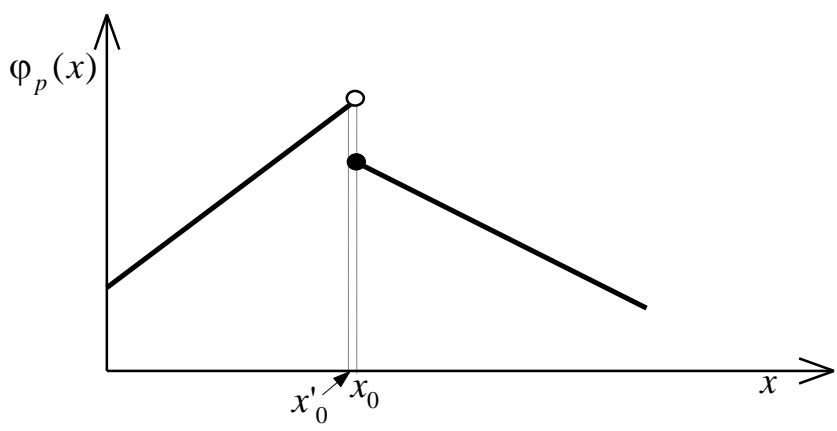

Figure 1 - Illustration of a case where the pessimistic solution does not exist

In addition to the optimistic and pessimistic solutions, other types of solutions can be defined. These solutions offer useful information to the leader's decision process on possible outcomes and ranges of values resulting from different decisions in an SVBP: the result of a failed optimistic approach - deceiving solution - and the result of a successful pessimistic approach - rewarding solution (Alves and Antunes, 2018a) (Alves, Antunes and Costa, 2019).

Definition 4 - Deceiving solution

Let $\left(x^{\mathrm{o}}, y^{\mathrm{o}}\right)$ be an optimistic solution. A deceiving solution, $\left(x^{\mathrm{d}}, y^{\mathrm{d}}\right)$, is obtained when the leader makes the optimistic decision $x^{\mathrm{o}}$ but the follower's reaction is the least favorable to the leader, i.e., $\left(x^{\mathrm{d}}, y^{\mathrm{d}}\right)=\left(x^{\mathrm{o}}, y^{\mathrm{d}}\right)$ where $y^{\mathrm{d}} \in \underset{y}{\arg \min }\left\{F\left(x^{\mathrm{o}}, y\right): y \in \Psi_{\mathrm{Ef}}\left(x^{\mathrm{o}}\right)\right\}$.

\section{Definition 5 - Rewarding solution}

Let $\left(x^{\mathrm{p}}, y^{\mathrm{p}}\right)$ be a pessimistic solution. A rewarding solution, $\left(x^{\mathrm{r}}, y^{\mathrm{r}}\right)$, is obtained when the leader makes a pessimistic decision $x^{\mathrm{p}}$ but the follower's reaction is the most favorable to the leader, i.e., $\left(x^{\mathrm{r}}, y^{\mathrm{r}}\right)=\left(x^{\mathrm{p}}, y^{\mathrm{r}}\right)$ where $y^{\mathrm{r}} \in \underset{y}{\arg \max }\left\{F\left(x^{\mathrm{p}}, y\right): y \in \Psi_{\mathrm{Ef}}\left(x^{\mathrm{p}}\right)\right\}$.

Therefore, according to the more optimistic or more pessimistic leader's attitude in the SVBP, the optimistic and pessimistic approaches can be distinguished, in which four extreme solutions may be identified: the optimistic solution, which is obtained when the follower decides in the most favorable way for the leader leading to his best possible objective function value; the deceiving solution, when the leader sets his variables $x$ embodying an optimistic attitude but the follower's choice is the worst for the leader; the pessimistic solution, in which the leader obtains his best objective function value when the follower's choice 
for each upper-level variables $x$ is the worst for the leader; the rewarding solution, when the leader adopts a pessimistic attitude setting his variables $x$ but the follower's reaction is the most favorable to the leader.

Let us define the optimistic frontier as the set of feasible solutions such that the follower's efficient solutions are the best for the leader and the pessimistic frontier as the set of feasible solutions such that the follower's efficient solutions are the worst for the leader.

Definition 6 - Optimistic frontier (Of)

The optimistic frontier is $\quad$ of $=\left\{\left(x, y^{\prime}\right) \in \mathbb{R}^{n_{1}} \times \mathbb{R}^{n_{2}}: G(x) \leq 0, y^{\prime} \in O(x)\right\} \quad$ with $O(x)=\left\{\underset{y}{\arg \max _{y}}\left\{F(x, y): y \in \Psi_{\mathrm{Ef}}(x)\right\}\right\}$.

Definition 7 - Pessimistic frontier (Pf)

$$
\begin{aligned}
& \text { The pessimistic frontier is } P f=\left\{\left(x, y^{\prime \prime}\right) \in \mathbb{R}^{n_{1}} \times \mathbb{R}^{n_{2}}: G(x) \leq 0, y^{\prime \prime} \in P(x)\right\} \text { with } \\
& P(x)=\left\{\underset{y}{\arg \min _{y}}\left\{F(x, y): y \in \Psi_{\mathrm{Ef}}(x)\right\}\right\} .
\end{aligned}
$$

The optimistic and the pessimistic solutions are the feasible solutions with maximum (best) value of $F$ in the optimistic frontier and in the pessimistic frontier, respectively. The optimistic and rewarding solutions belong to $O f$, while the pessimistic and deceiving solutions belong to $P f$.

Remark 1 - The value $F\left(x^{\mathrm{d}}, y^{\mathrm{d}}\right)$ of the deceiving solution can be worse and is never better than the value $F\left(x^{\mathrm{p}}, y^{\mathrm{p}}\right)$ of the pessimistic solution.

This remark is justified as follows. The pessimistic solution $\left(x^{\mathrm{p}}, y^{\mathrm{p}} \in P\left(x^{\mathrm{p}}\right)\right)$ is the best solution according to $F(x, y)$ on $P f$ (definition 3 ). The deceiving solution $\left(x^{\mathrm{d}}, y^{\mathrm{d}} \in P\left(x^{\mathrm{d}}\right)\right)$ also belongs to $P f$ (definition 4). Thus, the deceiving solution must satisfy $F\left(x^{\mathrm{d}}, y^{\mathrm{d}}\right) \leq F\left(x^{\mathrm{p}}, y^{\mathrm{p}}\right)$, otherwise it would be the pessimistic solution itself.

In order to illustrate these four solutions, let us consider the linear SVBP in Example 1, with one upperlevel variable $(x)$ and two lower-level variables ( $y_{1}$ and $\left.y_{2}\right)$ :

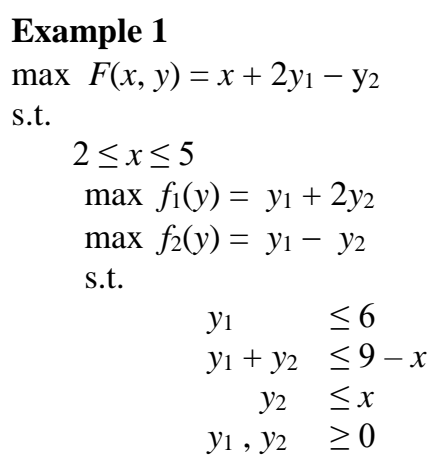

This problem is represented in Figure 2, where the set of all lower-level efficient solutions, $\Psi_{\mathrm{Ef}}(x)$ for all feasible $x$, is displayed by the darker faces. Figure 3 shows the $F$ values on $O f$ and on $P f$ of the linear SVBP of Figure 2.

In this example there is a significant difference between the optimistic and the deceiving solutions for the leader's objective function $F\left(F^{\mathrm{o}}=15\right.$ and $\left.F^{\mathrm{d}}=6\right)$, being the $F$ value in the rewarding solution $\left(F^{\mathrm{r}}=14\right)$ close to the optimistic $F$. Therefore, if the leader adopts an optimistic approach he takes a high risk, since the deceiving solution is significantly worse than the pessimistic one $\left(F^{\mathrm{p}}=10\right)$. This would not be the case if $F^{\mathrm{d}}$ was close to $F^{\mathrm{p}}$, in which the optimistic approach could be more attractive than the pessimistic one. 


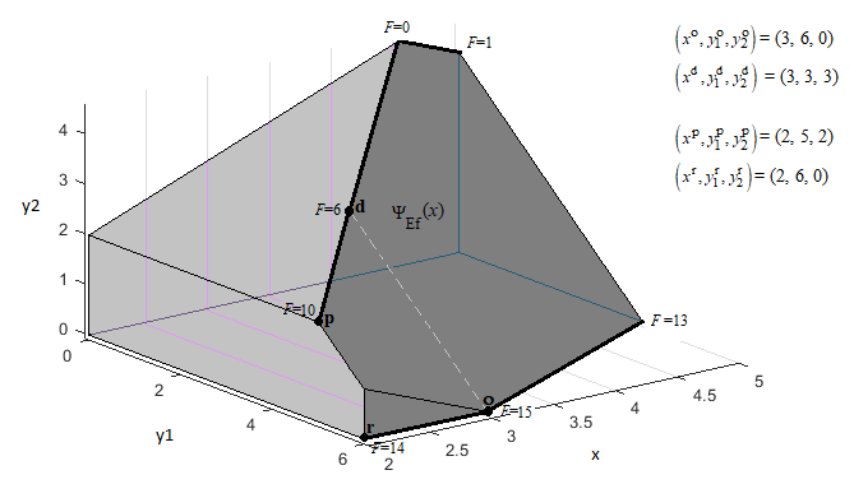

Figure 2 - Example 1 of a linear SVBP, showing the optimistic, pessimistic, deceiving and rewarding solutions in the decision variable space.

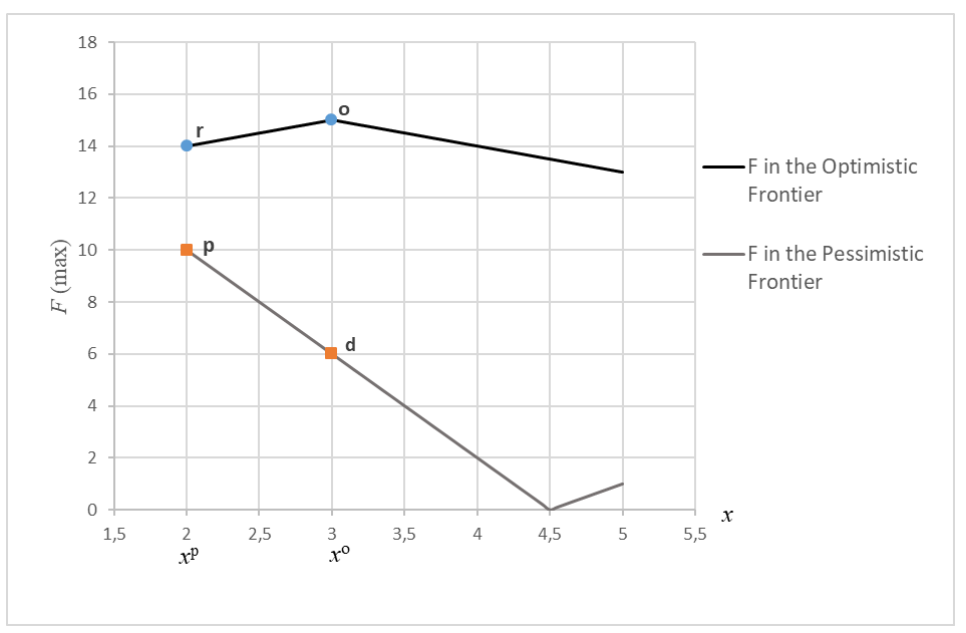

Figure $3-F$ values in the optimistic and pessimistic frontiers of Example 1.

\section{Dealing with the leader's decision risk in SVBP - moderate solutions}

In SVBP, the leader may take a high risk engaging in an optimistic attitude if the deceiving solution is significantly worse than the pessimistic one. If the leader adopts a pessimistic attitude, he still has the opportunity to obtain the rewarding solution, which may be close to the optimistic one, as in Example 1. Therefore, for decision support purposes, it is important to offer the leader other types of solutions besides the four extreme ones proposed above. These moderate solutions are more balanced regarding pure optimistic vs. pessimistic approaches, i.e., a moderate solution provides the highest expected value for $F(x, y)$ considering an optimism/pessimism index (e.g., probabilities of the follower's decision being in favor or against the interests of the leader).

Definition 8 - Moderate solution

Let $p_{1} \in[0,1]$ be an optimism index defined by the leader representing the probability perceived by leader for the follower choosing, for each $x$, the solution $y \in \Psi_{\mathrm{Ef}}(x)$ that is the best for the leader; $p_{2} \in[0,1], p_{2}=$ $\left(1-p_{1}\right)$, is the pessimism index (probability for the follower choosing, for each $x$, the solution $y \in \Psi_{E f}(x)$ that is the worst for the leader). The moderate solution, $\left(x^{\mathrm{m}}, y^{\mathrm{m}}\right)$, is the one that optimizes the expected value problem (EVP):

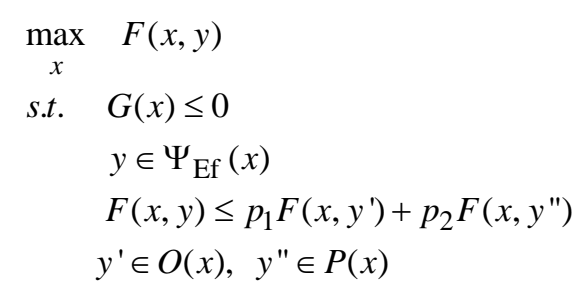


In practice, the values of $p_{1}\left(p_{2}\right)$ may be derived from the experience of the leader regarding the follower's reaction to his decisions. Moreover, solving the expected value problem for different values that cover the whole range of $p_{1}\left(p_{2}\right)$ offers the leader an overview of the variation of $F$, and then information enabling to hedge against (different probabilities) of the follower's decision being in favor or against the interests of the leader.

Other approaches may involve eliciting the follower's preferences, for instance using aspiration/reservation levels for the objective function values or weighting techniques. Solutions to SVBP/MOBP for different preference settings provide relevant information regarding the balance risk vs. opportunity associated with each leader's decision vis-à-vis the follower's possible choices.

Associated with the moderate solution, we can define for the same leader's decision $\left(x^{\mathrm{m}}\right)$ the solutions obtained on the optimistic frontier and on the pessimistic frontier: $\left(x^{\mathrm{m}}, y^{\prime}\right)$ with $y^{\prime} \in O\left(x^{\mathrm{m}}\right)$ and $\left(x^{\mathrm{m}}, y^{\prime \prime}\right)$ with $y^{\prime \prime} \in P\left(x^{\mathrm{m}}\right)$. These can be seen as the rewarding and the deceiving solutions for a moderate approach for a given $p_{1}$, which are also obtained when solving (EVP). These solutions may convey useful information to frame the possible outcomes for the leader if he takes that moderate decision.

Figure 4 shows, for Example 1, the lines of $F$ values for different values of $p_{1}$. The optimistic frontier corresponds to $p_{1}=1$ and the pessimistic frontier corresponds to $p_{1}=0$. In this example, for values of the optimism index $p_{1}$ from 1 to 0.8 , the best leader's decision $x$ according to the (EVP) formulation is the optimistic one, $x^{\mathrm{o}}$; for $p_{1}=0.8$, the $F$ expected value is equal for the leader's decisions $x^{\mathrm{o}}$ and $x^{\mathrm{p}}$; for $p_{1}<0.8$, the best leader's decision $x$ according to (EVP) is the pessimistic one, $x^{\mathrm{p}}$. This information complements the analysis above of the four extreme solutions concerning the assessment of the leader's risk associated with his decisions.

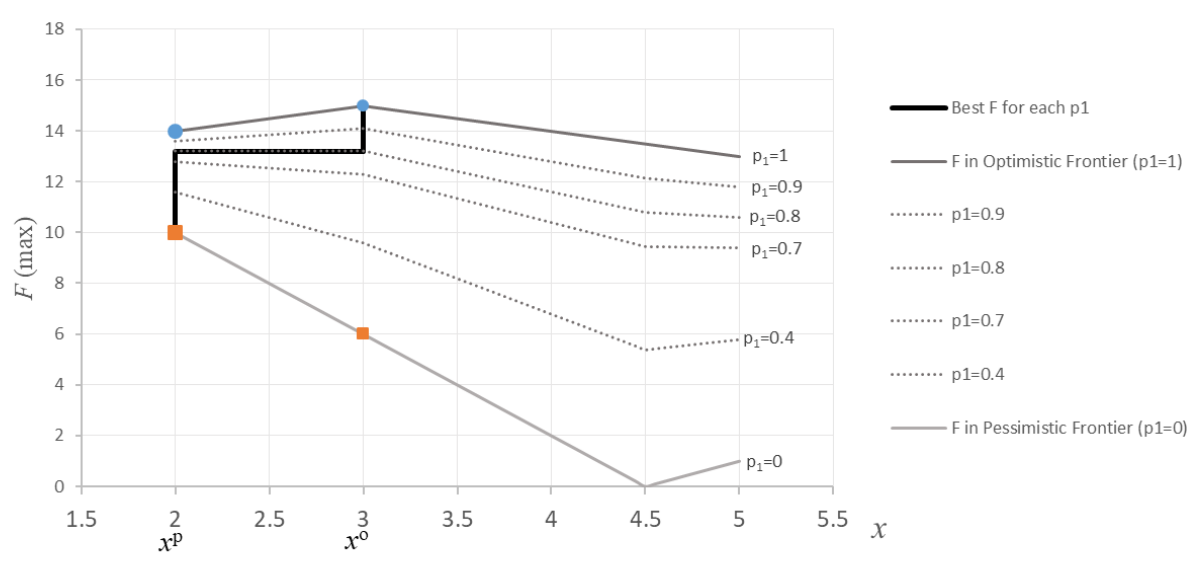

Figure 4 - Example 1: $F$ values for different $p_{1}$ values and the best leader's option for each one.

Example 2 refers to a nonlinear SVBP with all objective functions to be minimized. This problem is adapted from a MOBP in (Deb and Sinha, 2009) considering only the first upper-level objective function.

\section{Example 2}

$$
\begin{array}{ll}
\min _{x} & F(x, y)=\left(y_{1}-1\right)^{2}+y_{2}^{2}+x^{2} \\
\text { s.t. } & \min _{y} f_{1}(x, y)=y_{1}^{2}+y_{2}^{2} \\
& \min _{y} f_{2}(x, y)=\left(y_{1}-x\right)^{2}+y_{2}^{2} \\
& \text { s.t. } \quad-1 \leq y_{1}, y_{2}, x \leq 2
\end{array}
$$

For a given value of $x$, the efficient solutions $\Psi_{\mathrm{Ef}}(x)$ to the lower-level problem are:

$$
\left(y_{1}, y_{2}\right) \in \mathbb{R}^{2}: \begin{cases}y_{1} \in[0, x], y_{2}=0 & \text { for } 0 \leq x \leq 2 \\ y_{1} \in[x, 0], y_{2}=0 & \text { for }-1 \leq x \leq 0\end{cases}
$$




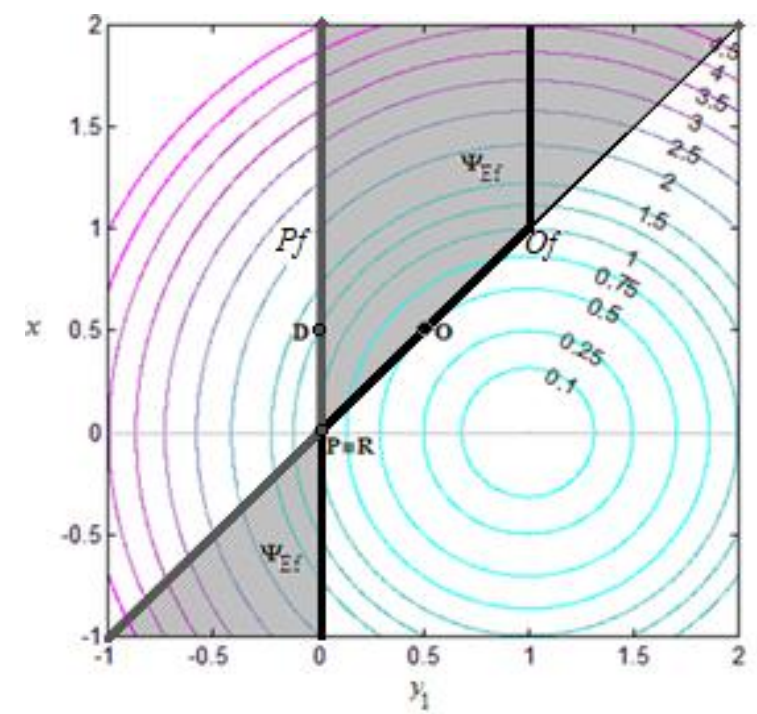

Figure 5 - Example 2: optimistic (O), pessimistic (P), deceiving (D) and rewarding (R) solutions

In Figure 5, the shaded area $\Psi_{\mathrm{Ef}}$ represents the induced region, i.e. $\Psi_{\mathrm{Ef}}(x)$ for all $x$. The level curves of $F$ $(x, y)$ are circles centered at the point $\left(y_{1}, x\right)=(1,0)$. Figure 5 displays only the variables $y_{1}$ and $x$ because $y_{2}$ $=0$ for all efficient solutions to the lower-level problem. This graph also shows the optimistic and pessimistic frontiers ( $O f$ and $P f$ ). Note that, for each $x$ other than $x=2$, only one point belongs to each frontier; for $x=2$, Of also has only one point $\left(y_{1}=1\right)$ but two points belong to $\operatorname{Pf}\left(y_{1}=0\right.$ and $\left.y_{1}=2\right)$, because there are two efficient solutions to the lower-level problem that provide the worst value for the leader $(F=5)$.

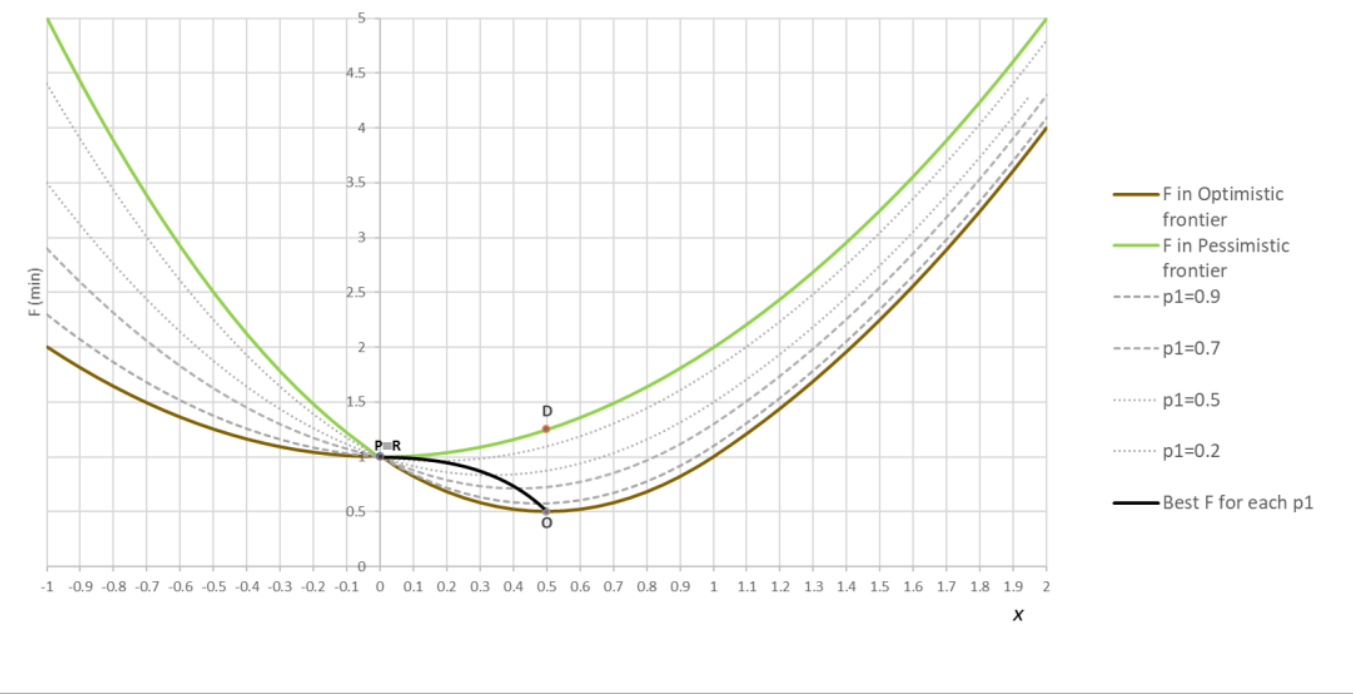

Figure 6 - Example 2: $F$ values on the Optimistic frontier, Pessimistic frontier and on the frontiers for different $p_{1}$ values; the best leader's option for each $p_{1}$.

Figure 6 shows, for Example 2, the curves of $F$ values for different values of the optimism index $p_{1}$. The best $F$ value for each $p_{1}$ is depicted in the solid black line. In this nonlinear example, each different $p_{1}$ leads to a different leader's best decision $x$ according to the EVP, i.e., to different moderate solutions. Unlike in Example 1, the leader's decision that provides the best expected value is not only the optimistic or the pessimistic solution, but rather a continuous range of values from $x^{0}$ to $x^{\mathrm{p}}$ that vary with $p_{1}$.

Figure 7 shows the $F$ value as function of the optimism index. For instance, for $p_{1}=0.5$, the moderate solution gives an $F$ value to the leader slightly over 0.8 . We can see this value in the two graphs of Figure 7; it is possible to determine analytically that the exact value of $F$ is 0.833 .

To sum up, different types of solutions can provide broader information to support the leader's decision process in SVBP. The optimistic, pessimistic, deceiving and rewarding solutions characterize distinct 
attitudes of the leader and the follower, delimiting the ranges of possible optimal values for the leader considering the follower's extreme decisions. Further information is offered by moderate solutions, which optimize the expected value for the leader's objective function considering different probabilities of the follower's decision being in favor or against the interests of the leader.

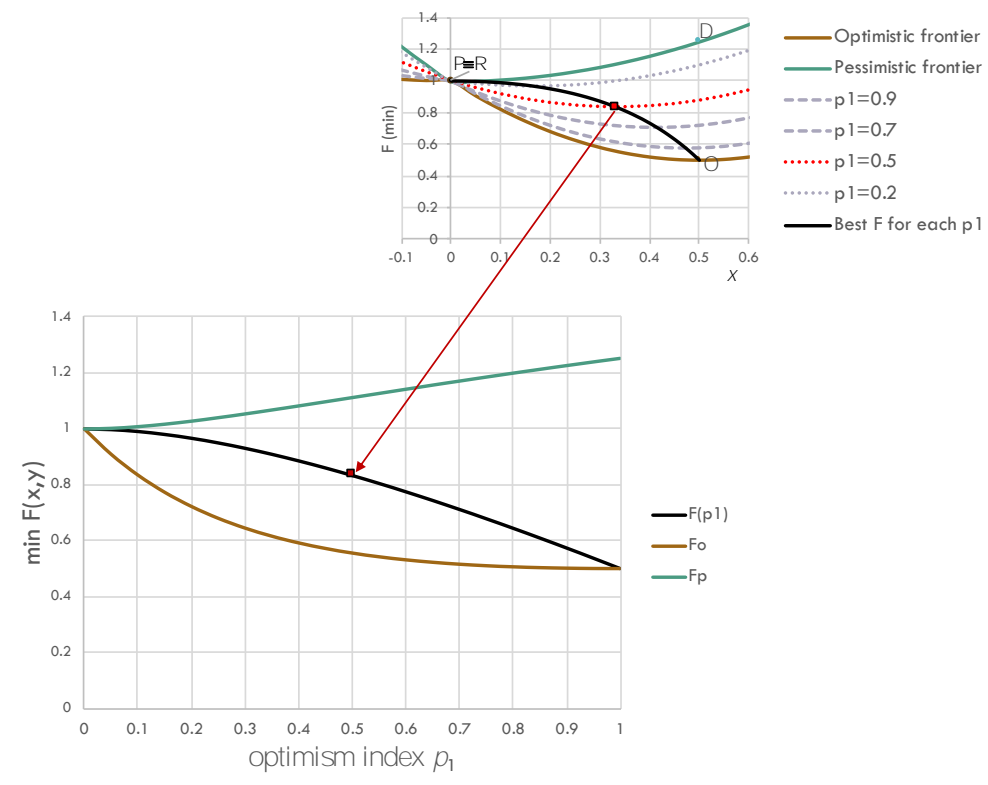

Figure 7 - Example 2: $F$ values value as function of the optimism index $p_{1}$.

\section{Optimistic and Pessimistic Pareto Fronts to the MOBP}

Concerning the bilevel problem with multiple objective functions at both levels, the aim of most procedures referred to in the literature has been to approximate the whole upper-level optimistic Pareto front or they consider that the follower's preferences are known, thus reducing the MOBP to a BP with multiple objective functions at the upper-level and a single objective function at the lower-level.

Recall the definition of induced region: $I R=\left\{(x, y) \in \mathbb{R}^{n_{1}} \times \mathbb{R}^{n_{2}}: x \in X, y \in \Psi_{\mathrm{Ef}}(x)\right\}$.

Let $\boldsymbol{F}(x, y)$ denote the objective vector $\left(F_{1}(x, y), \ldots, F_{k}(x, y)\right)$. A solution $(x, y)$ dominates $\left(x^{\prime}, y^{\prime}\right)$ according to the upper-level objective functions, $\boldsymbol{F}(x, y) \succ \boldsymbol{F}\left(x^{\prime}, y^{\prime}\right)$, if $F_{j}(x, y) \geq F_{j}\left(x^{\prime}, y^{\prime}\right)$ for all $j=1, \ldots, k$, and $F_{j}(x, y)>F_{j}\left(x^{\prime}, y^{\prime}\right)$ for at least one $j$.

Definition 9 - Optimistic F-efficient solution

A feasible solution $\left(x^{\prime}, y^{\prime}\right) \in I R$ to the MOBP is optimistic F-efficient if there is no other $(x, y) \in I R$ that dominates $\left(x^{\prime}, y^{\prime}\right)$, i.e. such that $\boldsymbol{F}(x, y) \succ \boldsymbol{F}\left(x^{\prime}, y^{\prime}\right)$.

This definition assumes an optimistic approach because it presumes that the leader can take any solution of $I R$ or, in other words, the follower only selects $f$-efficient solutions to his problem that are also efficient for the leader, thus leading to a subset of $I R$ containing the best solutions to the leader, i.e., all his efficient solutions.

The Optimistic Pareto Front $(O P F)$ to the MOBP is composed by all optimistic $F$-efficient solutions $(x, y)$.

The existing algorithms that aim to approximate the $O P F$ (e.g. (Deb and Sinha, 2010), (Zhang et al., 2013), (Carrasqueira, Alves and Antunes, 2015)) employ the following general scheme: an upper-level search is performed for different $x$; for each $x^{\prime} \in X$, a lower-level search approximates $\Psi_{\mathrm{Ef}}\left(x^{\prime}\right)$ by keeping an temporary archive $A\left(x^{\prime}\right)$ of solutions $\left(x^{\prime}, y\right)$ that do not dominate each other according to the lower-level objective functions $f$; once the search for approximating $\Psi_{\mathrm{Ef}}\left(x^{\prime}\right)$ has been finished, the solutions $\left(x^{\prime}, y\right) \in$ 
$A\left(x^{\prime}\right)$ are compared with the solutions $(x, y)$ in an external archive $A$ such that only solutions that are not dominated by any other according to the upper-level objective functions $F$ remain in $A$. At the end of the algorithm, $A$ is the approximation of the $O P F$. We can observe that, although the concept of optimistic Pareto optimal ( $F$-efficient) solution to a MOBP is well defined, it can be very difficult to implement a procedure to compute solutions in the $O P F$ using this scheme, even if the aim is just to compute some solutions, for two main reasons:

- $\quad$ the algorithm should ensure that the lower-level solutions $y$ (for a given $x$ ) are $f$-efficient; otherwise they are not feasible to the MOBP;

- $\quad$ to guarantee that optimistic $F$-efficient solutions $(x, y)$ are yielded, the algorithm should ensure that no other $x^{\prime} \in X$ exists such that some $\left(x^{\prime}, y^{\prime}\right)$, with $y^{\prime}$ being $f$-efficient, dominates $(x, y)$.

Similar concepts associated with a pessimistic approach are more difficult to define in bilevel problems with multiple objective functions at both levels, and they may not be consensual. Let us consider the following principle: for a given $x$, the follower's decisions that are the worst for the leader are the solutions in $\Psi_{\mathrm{Ef}}(x)$ that are the "most dominated" ones for the leader, if different ranks of dominance (for the upper-level) exist among the solutions in $\Psi_{\mathrm{Ef}}(x)$. For instance, consider three $f$-efficient solutions for the same $x$, $\left(x, y^{1}\right),\left(x, y^{2}\right),\left(x, y^{3}\right)$, with $y^{1}, y^{2}, y^{3} \in \Psi_{\mathrm{Ef}}(x)$, and $\boldsymbol{F}\left(x, y^{1}\right) \prec \boldsymbol{F}\left(x, y^{2}\right) \prec \boldsymbol{F}\left(x, y^{3}\right)$, where $a \prec b$ denotes that $a$ is dominated by $b$. Then, a pessimistic approach may assume that the follower chooses $y^{1}$, the worst option for the leader. Therefore, for each $x$, the solutions in $\Psi_{\mathrm{Ef}}(x)$ that do not dominate any other in $\Psi_{\mathrm{Ef}}(x)$ are retrieved as the pessimistic ones.

Definition 10 - "Most dominated" solutions for the leader

A lower level efficient solution $y^{\prime} \in \Psi_{\mathrm{Ef}}\left(x^{\prime}\right)$ is said to be "most dominated" for the leader, i.e., worst for the leader, for a given upper level solution $x^{\prime}$, if there is no other $y \in \Psi_{\mathrm{Ef}}\left(x^{\prime}\right)$ that is dominated by $y^{\prime}$ according to the upper level objective functions, i.e., such that $\left.\boldsymbol{F}\left(x^{\prime}, y\right) \prec \boldsymbol{F}\left(x^{\prime}, y^{\prime}\right)\right\}$.

Definition 11 - Induced pessimistic region

Let $\Psi_{\text {Ef-worstF }}(x)=\left\{y^{\prime} \in \Psi_{\mathrm{Ef}}(x)\right.$ : there is no other $y \in \Psi_{\mathrm{Ef}}(x)$ such that $\left.\boldsymbol{F}(x, y) \prec \boldsymbol{F}\left(x, y^{\prime}\right)\right\}$ denote the set of all "most dominated" solutions for the leader for each $x \in X$. The induced pessimistic region to the MOBP is $I R_{\text {worstF }}=\left\{(x, y) \in \mathbb{R}^{n_{1}} \times \mathbb{R}^{n_{2}}: x \in X, y \in \Psi_{\text {Ef-worstF }}(x)\right\}$.

\section{Definition 12 - Pessimistic F-efficient solution}

A feasible solution $\left(x^{\prime}, y^{\prime}\right) \in I R_{\mathrm{worstF}}$ to the MOBP is pessimistic F-efficient if there is no other $(x, y) \in I R_{\text {worstF }}$ that dominates $\left(x^{\prime}, y^{\prime}\right)$, i.e. such that $\boldsymbol{F}(x, y) \succ \boldsymbol{F}\left(x^{\prime}, y^{\prime}\right)$.

The Pessimistic Pareto Front $(P P F)$ to the MOBP is composed by all pessimistic F-efficient solutions $(x, y)$.

The following MOBP examples illustrate these concepts.

Let us first consider the nonlinear problem presented in Example 2 with a second upper-level objective function (problem 3 in (Deb and Sinha, 2009)).

\section{Example 3}

$$
\begin{array}{ll}
\min _{x} & F_{1}(x, y)=\left(y_{1}-1\right)^{2}+y_{2}^{2}+x^{2} \\
\min _{x} & F_{2}(x, y)=\left(y_{1}-1\right)^{2}+y_{2}^{2}+(x-1)^{2} \\
\text { s.t. } & \min _{y} f_{1}(x, y)=y_{1}^{2}+y_{2}^{2} \\
& \min _{y} f_{2}(x, y)=\left(y_{1}-x\right)^{2}+y_{2}^{2} \\
& \text { s.t. } \quad-1 \leq y_{1}, y_{2}, x \leq 2
\end{array}
$$


The lower-level efficient solutions are the same as in Example 2, represented by the shaded area in Figure 8. In all lower-level efficient solutions, $y_{2}=0$. The level curves of $F_{1}(x, y)$ and $F_{2}(x, y)$ are circles centered at the points $\left(y_{1}, x\right)=(1,0)$ and $\left(y_{1}, x\right)=(1,1)$, respectively. If separately considered, the objective functions $F_{1}(x, y)$ and $F_{2}(x, y)$ have the same optimistic frontier $(O f)$ and the same pessimistic frontier $(P f)$ for the corresponding SVBL problems. $O f$ and $P f$ are represented in Figure 8. Therefore, the $O P F$ to the MOBP is located on the common $O f$ of $F_{1}(x, y)$ and $F_{2}(x, y)$ between their individual optima $\left(\mathrm{O}_{1}\right.$ and $\left.\mathrm{O}_{2}\right)$.

$\mathrm{O}_{1}:\left(x, y_{1}, y_{2}\right)=(0.5,0.5,0),\left(f_{1}, f_{2}\right)=(0.25,0),\left(F_{1}, F_{2}\right)=(0.5,0.5)$.

$\mathrm{O}_{2}:\left(x, y_{1}, y_{2}\right)=(1,1,0),\left(f_{1}, f_{2}\right)=(1,0),\left(F_{1}, F_{2}\right)=(1,0)$.

Pf indicates the subset of the follower's efficient solutions that are "worst for the leader". Therefore, the $P P F$, according to the above definition for this MOBP, is located on the common $P f$ between the individual pessimistic solutions $\left(\mathrm{P}_{1}\right.$ and $\left.\mathrm{P}_{2}\right)$ to $F_{1}(x, y)$ and $F_{2}(x, y)$.

$\mathrm{P}_{1}:\left(x, y_{1}, y_{2}\right)=(0,0,0),\left(f_{1}, f_{2}\right)=(0,0),\left(F_{1}, F_{2}\right)=(1,2)$.

$\mathrm{P}_{2}:\left(x, y_{1}, y_{2}\right)=(1,0,0),\left(f_{1}, f_{2}\right)=(0,1),\left(F_{1}, F_{2}\right)=(2,1)$.

Thus, $O P F$ is obtained for $x \in[0.5,1], y_{1}=x, y_{2}=0 ; P P F$ is obtained for $x \in[0,1], y_{1}=y_{2}=0$.

Note that, although this example is nonlinear, it is easier to analyze than a general MOBP problem, due to the fact that $O f$ and $P f$ are coincident for $F_{1}$ and $F_{2}$ when considered separately. That is, for each $x$, the lowerlevel efficient solution $y^{\prime} \in \Psi_{\mathrm{Ef}}(x)$ that is the best for $F_{1}$ is also the best for $F_{2}$ and $y^{\prime \prime} \in \Psi_{\mathrm{Ef}}(x)$ that is the worst for $F_{1}$ is also the worst for $F_{2}$. As a result, there is one solution for each $x$ that dominates all the others w.r.t. the upper-level objective functions and there is only one solution that is the "most dominated".

Figure 9 shows the (coincident) $O f$ and $P f$ of $F_{1}$ and $F_{2}$, as well $O P F$ and $P P F$ in the upper-level objective function space.

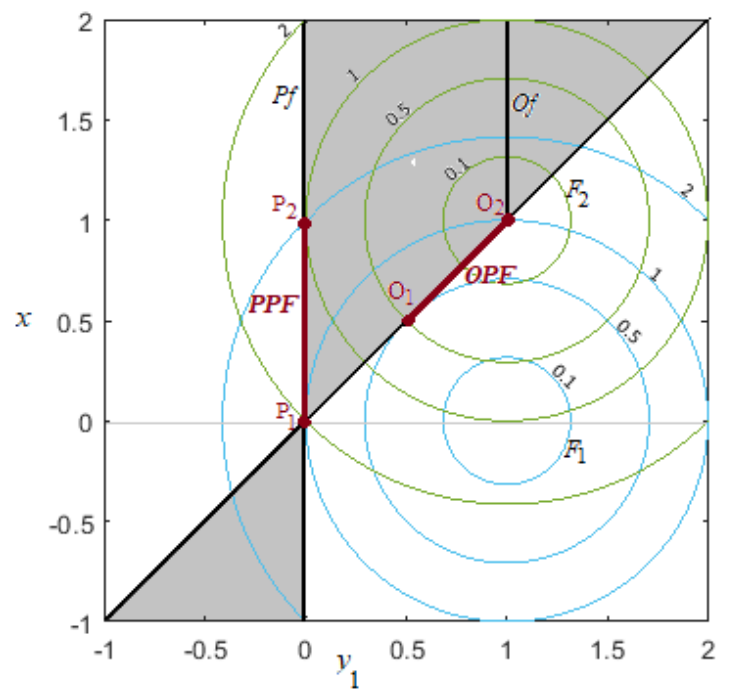

Figure 8 - Example 3: Optimistic Pareto Front $(O P F)$ and Pessimistic Pareto Front $(P P F)$ in the variable space 


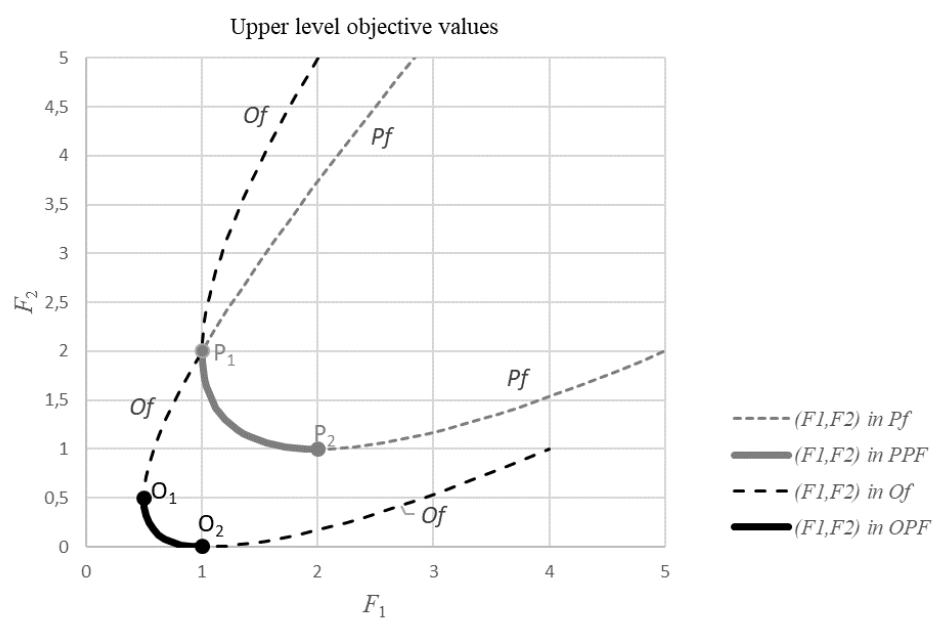

Figure 9 - Values of the upper-level objective functions in the Optimistic Pareto Front $(O P F)$ and in Pessimistic Pareto Front $(P P F)$

Remark 2. Nondominated solutions according to the upper-level objective functions within each $\Psi_{\mathrm{Ef}}(x)$ are not necessarily efficient solutions to the leader.

This remark can be easily shown using the previous example. Consider, for instance, $x=2$ : for this value of $x$, there is one solution $(2, y)$ that dominates all the others $\left(2, y^{\prime}\right)$ with $y, y^{\prime} \in \Psi_{\mathrm{Ef}}(2)$ according to the upperlevel objective functions; this nondominated solution within $\Psi_{\mathrm{Ef}}(2)$ is $\left(x, y_{1}, y_{2}\right)=(2,1,0)$ belonging to $O f$, where $\left(F_{1}, F_{2}\right)=(4,1)$. However, considering $x=1$, the solution $\left(x, y_{1}, y_{2}\right)=(1,1,0)$ has $\left(F_{1}, F_{2}\right)=(1,0)$, which dominates the previous solution. The point $(1,1,0)$ also belongs to $O f$ (point $\mathrm{O}_{2}$ in Fig. 8), i.e., it is a nondominated solution within $\Psi_{\mathrm{Ef}}(1)$. We can further observe that all solutions within $\Psi_{\mathrm{Ef}}(1)$ dominate $(x$, $\left.y_{1}, y_{2}\right)=(2,1,0)$ even the "most dominated" one: $\left(x, y_{1}, y_{2}\right)=(1,0,0)$, point $\mathrm{P}_{2}$ in Fig. 8 , with $\left(F_{1}, F_{2}\right)=(2$, $1)$, dominates $\left(x, y_{1}, y_{2}\right)=(2,1,0)$, with $\left(F_{1}, F_{2}\right)=(4,1)$ on the edge of $O f$. This can also be observed in Fig. 9.

Therefore, solutions that are nondominated within each $\Psi_{\mathrm{Ef}}(x)$ may be not efficient to the leader, considering optimistic $F$-efficiency or even pessimistic $F$-efficiency.

Remark 3. $O P F$ and $P P F$ are not necessarily disjoint, i.e., there may exist solutions that belong to both $O P F$ and $P P F$.

This remark will be shown in the next MOBP example, which further illustrates the difficulties when $O f$ and $P f$ are not coincident for $F_{1}$ and $F_{2}$.

For the sake of clarity in the graphical visualization, we consider a MOBP with linear objective functions and constraints and one upper-level integer variable.

\section{Example 4}

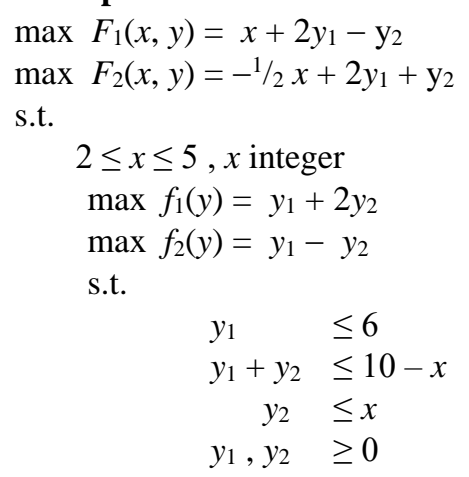


This problem is represented in Figure 10. In Figure 10(a) the constraint region with continuous $x$ is displayed; Figure 10(b) represents the constraint region for $x$ integer, the case considered in Example 4, where the set of all lower-level efficient solutions $\Psi_{\mathrm{Ef}}(x)$ for each $x=2, \ldots, 5$ is highlighted by darker thick lines. Figure 11 shows separate graphs for each $x$. This figure distinguishes the subset of solutions within each $\Psi_{\mathrm{Ef}}(x)$ that are dominated for the leader (dashed lines) from those that are nondominated (in solid black) which are: the line segments $[\mathrm{AB}]$ for $x=2$ and $[\mathrm{CD}]$ for $x=3$; the vertices $\mathrm{E}$ for $x=4$ and $\mathrm{F}$ for $x=5$. The corresponding upper-level objective values $\left[F_{1}, F_{2}\right]$ are displayed next to each vertex.

Although nondominated solutions within a $\Psi_{\mathrm{Ef}}(x)$ may be not efficient (i.e., they are not really nondominated) for the leader, as stated in Remark 2, for the sake of simplicity of the language, we will simply use the term non-dominated to designate solutions $(x, y)$ for which there is no other $\left(x, y^{\prime}\right), y^{\prime} \in \Psi_{\mathrm{Ef}}(x)$ that dominates it. As can be seen in Fig. 10 and Fig. 11, only for $x=4$ and $x=5$ a single non-dominated solution exists among $\Psi_{\mathrm{Ef}}(x)$ for the upper-level objective functions (points $\mathrm{E}$ and $\mathrm{F}$, respectively), a similar situation to the one observed in the Example 3 for every $x$. For $x=2$, all $\Psi_{\mathrm{Ef}}(2)$ are non-dominated for the leader, and for $x=3$ there are non-singleton subsets of $\Psi_{\mathrm{Ef}}(3)$ with dominated and non-dominated solutions for the leader. Thus, when separately considered, the objective functions $F_{1}$ and $F_{2}$ do not have the same $O f$ and $P f$.

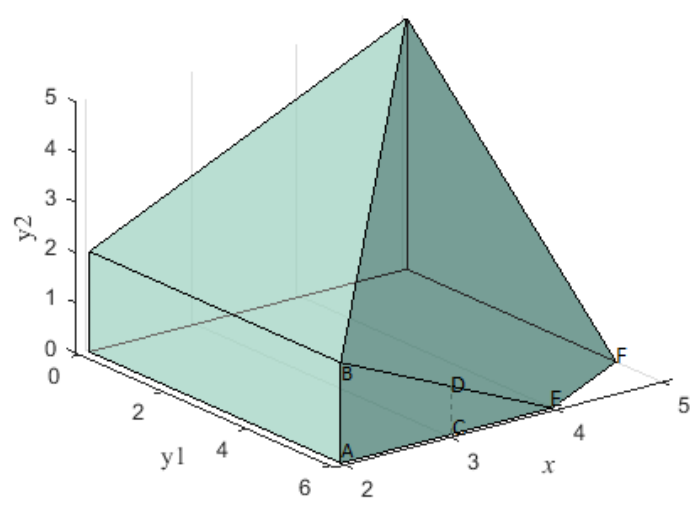

(a) $x$ continuous

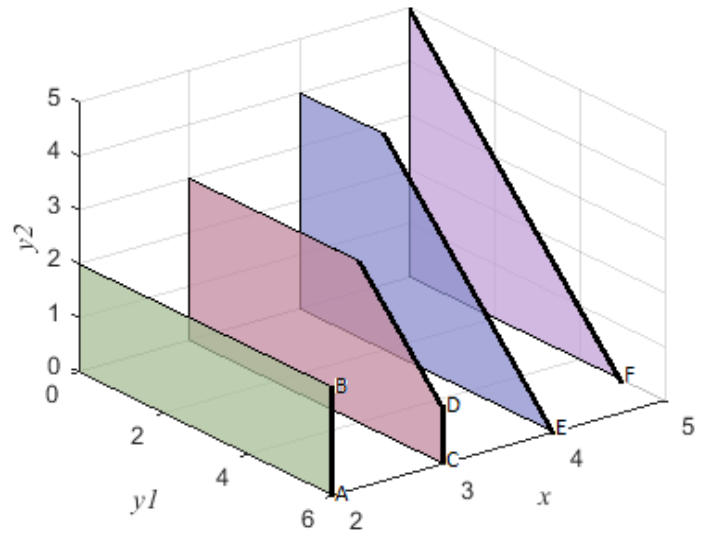

(b) $x$ integer

Figure 10 - Representation of the constraint region of Example 4.
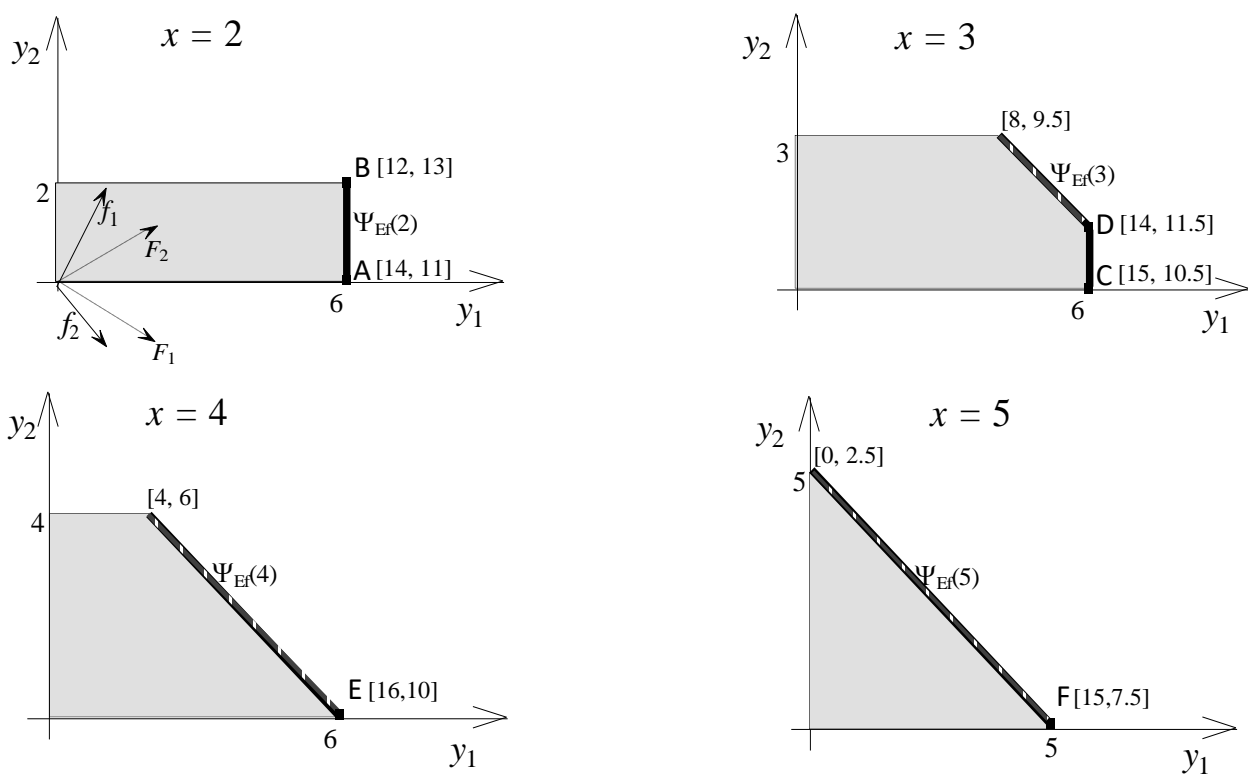

Figure 11 - Representation of Example 4 for each $x=2,3,4$, 5. For each vertex of $\Psi_{\mathrm{Ef}}(x)$, the upper-level objective values $\left[F_{1}, F_{2}\right]$ are displayed. 


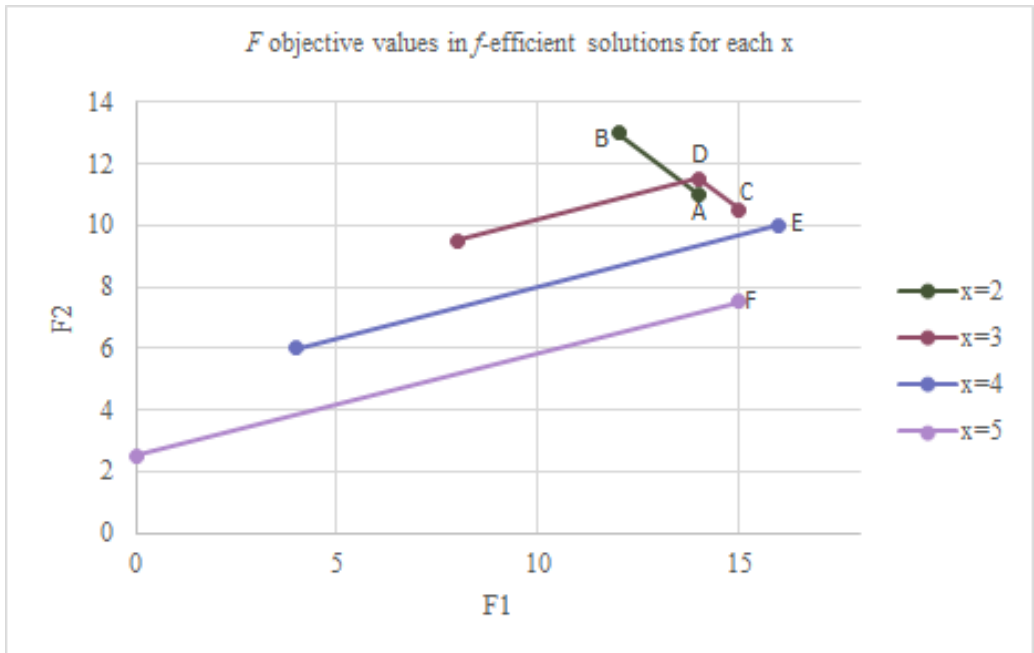

Figure 12 - Upper-level objective values in the lower-level efficient solutions for each $x$.

In Figure 12, the values of the upper-level objective functions $F_{1}$ and $F_{2}$ in all solutions of $\Psi_{\mathrm{Ef}}(x)$ for each $x$ are displayed, i.e. solutions in $I R$.

Figure 13(a) shows the values of $F_{1}$ and $F_{2}$ in $\Psi_{\mathrm{Ef}}(x)$ that are the best solutions for the leader (i.e. nondominated solutions for the leader within $\Psi_{\mathrm{Ef}}(x)$ ): [AB], [CD], E and F in Figures 12 and 13. The OPF (Fig. $13 \mathrm{~b})$ results from selecting the solutions that do not dominate each other among the best solutions for the leader (Fig. 13a); this consists of selecting the solutions that do not dominate each other among the whole $I R$ (Fig. 12). In Figure 13(b), the point $\mathrm{A}^{\prime}$ with $\left(F_{1}, F_{2}\right)=(13.5,11.5)$ is weakly efficient.

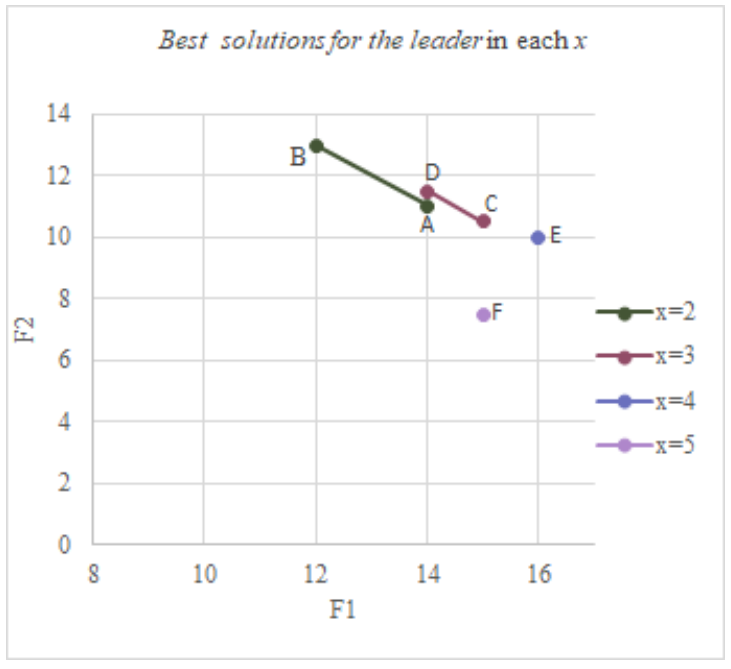

(a)

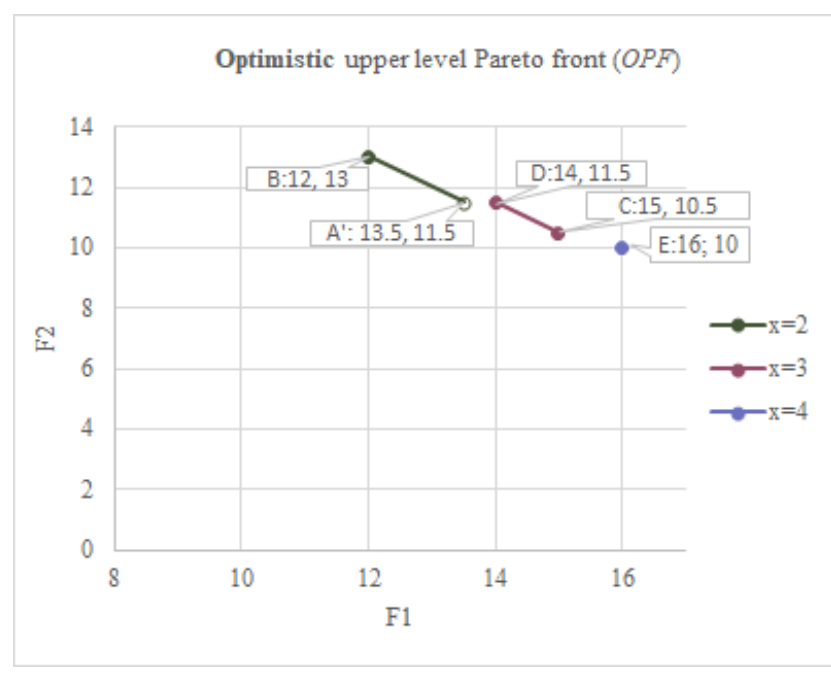

(b)

Figure 13 - Best solutions for the leader among the lower-level efficient solutions for each leader's decision (a) and the Optimistic Pareto Front (b).

Let us now suppose that the leader adopts a pessimistic approach. In this case, the leader assumes that the follower will choose a solution from the worst solutions for the leader in $\Psi_{\mathrm{Ef}}(x)$. As can be observed in Figure 12, for $x=2$ there is no solution in $\Psi_{\mathrm{Ef}}(2)$ that dominates another one according to the upper-level objective functions. Therefore, all of them are retrieved. For $x=3$, the worst solution for the leader is the one with $\left(F_{1}, F_{2}\right)=(8,9.5)$. For $x=4$ and $x=5$, there is also one point in each $\Psi_{\mathrm{Ef}}(x)$ that is the worst for the leader: $\left(F_{1}, F_{2}\right)=(4,6)$ and $(0,2.5)$, respectively. Figure 14(a) shows the values of $F_{1}$ and $F_{2}$ in the worst 
solutions for the leader for each $x\left(I R_{\mathrm{worstF}}\right)$ and Figure $14(\mathrm{~b})$ shows the $P P F$, which results from selecting the solutions that do not dominate each other among the solutions in $I R_{\mathrm{worstF}}$. Note that $P P F=[\mathrm{BA}]$, which includes part of $O P F\left(\left[\mathrm{BA}^{\prime}[)\right.\right.$ and other solutions that are dominated by solutions in $O P F$. The solutions in $P P F$ that do not belong to $O P F$ are $\left[\mathrm{A}^{\prime} \mathrm{A}\right]$, where $\mathrm{A}^{\prime}\left(\right.$ Fig.13b) has $\left(F_{1}, F_{2}\right)=(13.5,11.5)$ and A (Fig. 13a) has $\left(F_{1}, F_{2}\right)=(14,11)$.

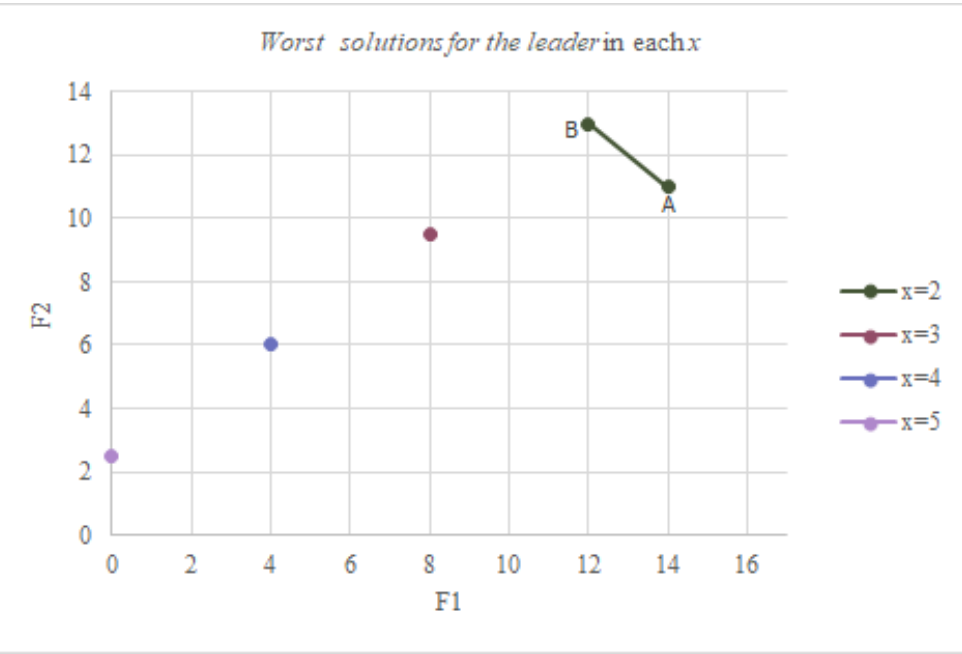

(a)

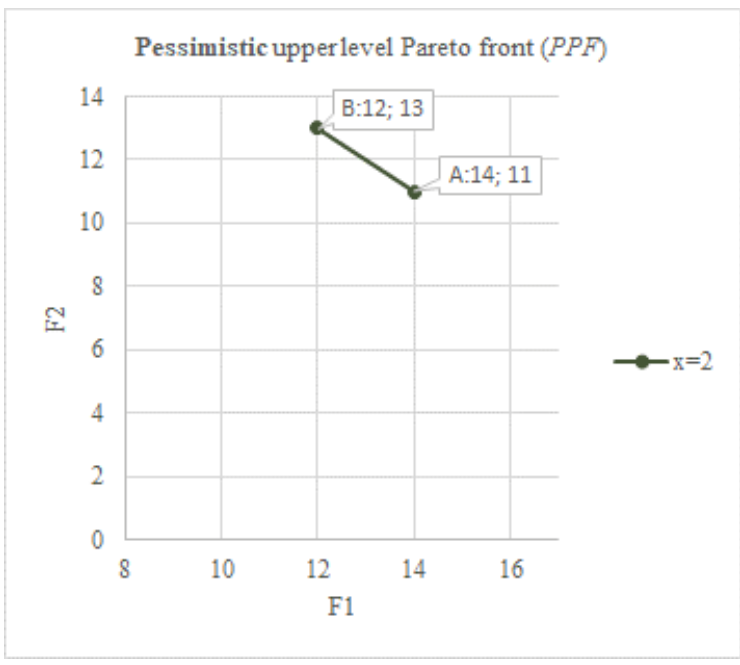

(b)

Figure 14 - Worst solutions for the leader among the lower-level efficient solutions for each leader's decision (a) and the Pessimistic Pareto Front (b).

The $P P F$ may be very difficult to compute, even more than the $O P F$, because it requires to isolate the solutions belonging to $I R_{\text {worstF }}$ and to select the non-dominated solutions within this set (and only within this set). Solutions in $I R_{\text {worstF }}$ may be difficult to identify when the worst solutions in $\Psi_{\mathrm{Ef}}(x)$ for each $F_{j}, j=1, . ., k$ do not coincide (i.e., the $P f$ for each $F_{j}$ are different): in order that a feasible solution can enter into $I R_{\text {worstF }}$, it must be assured that no other solution for the same $x$ is dominated by it according to the upper-level objective functions.

Finally, solutions belonging to both $O P F$ and $P P F$ may be interesting because they have associated low risk. The leader's decisions $x$ in a common set of $O P F$ and $P P F$ lead to efficient solutions to the leader regardless of the follower's choices.

\section{A Differential Evolution algorithm to compute the extreme and moderate solutions in SVBP}

The DE algorithm presented herein aims to compute the four extreme solutions and a moderate solution to the SVBP given an optimism index $p_{1}$ chosen by the leader.

The algorithm uses a population Pop of individuals, which is split into three sub-populations that share the same upper-level $x$ vectors: $P o p^{\prime}, P_{o p}{ }^{\prime}$ and $P_{o p}{ }^{+}$aiming at approximating, respectively, the optimistic frontier (where the optimistic and rewarding solutions will be searched for), the pessimistic frontier (for the pessimistic and deceiving solutions) and the frontier to search for the moderate solution, which we will call moderate frontier.

Let $\mathrm{Nu}$ be the number of upper-level individuals. Each sub-population has the following configuration: $\left\{\left(x_{1}, y_{1}\right),\left(x_{2}, y_{2}\right), \ldots,\left(x_{N u}, y_{N u}\right)\right\}$, where $y=y^{\prime}, y^{\prime \prime}$ or $y^{+}$, respectively in Pop', Pop" and Pop ${ }^{+}$.

DE operations are employed to evolve the population for the upper-level search and, for each vector $x_{i}$, $i=1, \ldots, N u$, a lower-level DE algorithm (LLROUTINE) is used to determine $\left(x_{i}, y_{i}^{\prime}\right),\left(x_{i}, y_{i}^{\prime \prime}\right)$ and $\left(x_{i}, y_{i}^{+}\right)$.

Considering the usual nomenclature of DE variants (Price, Storn and Lampinen, 2006), the DE variant $D E / \mathrm{rand} / 1 / \mathrm{bin}$ has been adopted for the upper-level search and $D E /$ best/1/bin has been used for the lowerlevel search, since this combination has revealed to have, in general, a better performance than the variants rand-rand or best-best in the two levels. For each individual $\pi_{i}$ (here generically representing an upper- 
level vector $x$ or a lower-level vector $y$ ), both variants randomly select two individuals $\pi_{r 1}, \pi_{r 2}$; DE/rand/1/bin also selects $\pi_{r 3}$ randomly, while $D E /$ best/1/bin assigns to $\pi_{r 3}$ the best individual in the current population. This latter variant is only used for the lower-level, where the best individual depends on whether the search is oriented towards the optimistic, pessimistic or moderate frontier respectively. In any variant, a trial vector $\mu_{i}$ is created as follows, where $D$ denotes the dimension of the vectors $\pi$ and $\mu$, $¥$ is the mutation scaling factor and CR is the crossover rate. We have used $¥=0.7$ and $\mathrm{CR}=0.9$, which are standard parameter values.

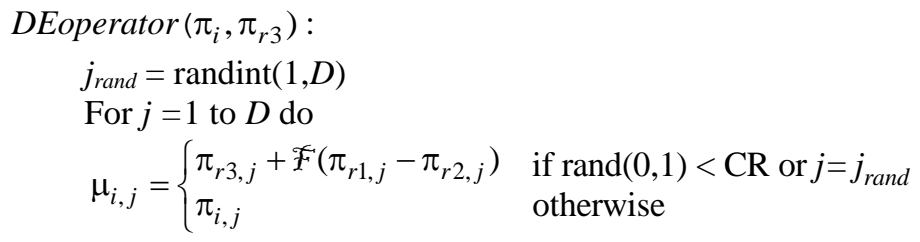

Concerning the satisfaction of constraints, if an upper-level solution does not satisfy the constraints, $x_{i} \notin X$, an attempt is made to repair it: if $X$ is a set of box constraints only, then each variable of $x$ is repaired by pulling it into its closest bound; if there are more general constraints difficult to meet, then the solution is penalized in the evaluation function $F$.

The output of the algorithm is: the optimistic solution $\left(x^{\mathrm{o}}, y^{\mathrm{o}}\right)$; the pessimistic solution $\left(x^{\mathrm{p}}, y^{\mathrm{p}}\right)$; the deceiving solution $\left(x^{\mathrm{d}}, y^{\mathrm{d}}\right)$; the rewarding solution $\left(x^{\mathrm{r}}, y^{\mathrm{r}}\right)$; and the moderate solution $\left(x^{\mathrm{m}}, y^{\mathrm{m}}\right)$. During the algorithm run these are incumbent solutions, which are initialized as follows:

$\left(x^{\mathrm{o}}, y^{\mathrm{o}}\right) \leftarrow \arg \max \left\{F(x, y):(x, y) \in\right.$ Pop $\left.^{\prime}\right\}$. Let $i 1$ be the index of $\left(x^{\mathrm{o}}, y^{\mathrm{o}}\right)$ in Pop';

$\left(x^{\mathrm{d}}, y^{\mathrm{d}}\right) \leftarrow\left(x_{i 1}, y_{i 1}^{\prime \prime}\right) \in$ Pop" $^{\prime \prime}$

$\left(x^{\mathrm{p}}, y^{\mathrm{p}}\right) \leftarrow \arg \max \left\{F(x, y):(x, y) \in P o p^{\prime \prime}\right\}$. Let $i 2$ be the index of $\left(x^{\mathrm{p}}, y^{\mathrm{p}}\right)$ in Pop";

$\left(x^{\mathrm{r}}, y^{\mathrm{r}}\right) \leftarrow\left(x_{i 2}, y_{i 2}^{\prime}\right) \in P o p^{\prime}$

$\left(x^{\mathrm{m}}, y^{\mathrm{m}}\right) \leftarrow \arg \max \left\{F(x, y):(x, y) \in\right.$ Pop $\left.^{+}\right\}$.

After the individuals have been changed, the incumbent solutions are updated. Let $u_{i}$ be a trial upper-level vector obtained from $x_{i}$ and let $\left(u_{i}, w_{i}^{\prime}\right),\left(u_{i}, w_{i}^{\prime \prime}\right),\left(u_{i}, w_{i}^{+}\right)$be the complete solutions corresponding to each sub-population obtained after the LLROUTINE (presented below in Algorithm 2) has been called. The optimistic / pessimistic / rewarding solution is updated, respectively with $\left(u_{i}, w_{i}^{\prime}\right) /\left(u_{i}, w_{i}^{\prime \prime}\right) /\left(u_{i}, w_{i}^{+}\right)$if this solution improves the corresponding incumbent solution. If the optimistic solution is updated with $\left(u_{i}, w_{i}^{\prime}\right)$ then the deceiving solution takes $\left(u_{i}, w_{i}^{\prime \prime}\right)$, and the reverse for the pessimistic/rewarding solutions.

The trial vector $u_{i}$ is accepted (ACCEPT function in Algorithm 1) when at least one of the solutions $\left(u_{i}, w_{i}\right)$ with $w_{i} \in\left\{w_{i}^{\prime}, w_{i}^{\prime \prime}, w_{i}^{+}\right\}$improves the value of $F$ with respect to the corresponding $\left(x_{i}, y_{i}\right)$ with $y_{i} \in\left\{y_{i}^{\prime}, y_{i}^{\prime \prime}, y_{i}^{+}\right\}$. If the trial vector is accepted, the three new solutions $\left(u_{i}, w_{i}\right), w_{i} \in\left\{w_{i}^{\prime}, w_{i}^{\prime \prime}, w_{i}^{+}\right\}$replace $\left(x_{i}, y_{i}\right), y_{i} \in\left\{y_{i}^{\prime}, y_{i}^{\prime \prime}, y_{i}^{+}\right\}$in $P o p^{\prime}, P o p^{\prime \prime}$ and $P o p^{+}$, respectively.

The general scheme for the upper-level search is described in Algorithm 1.

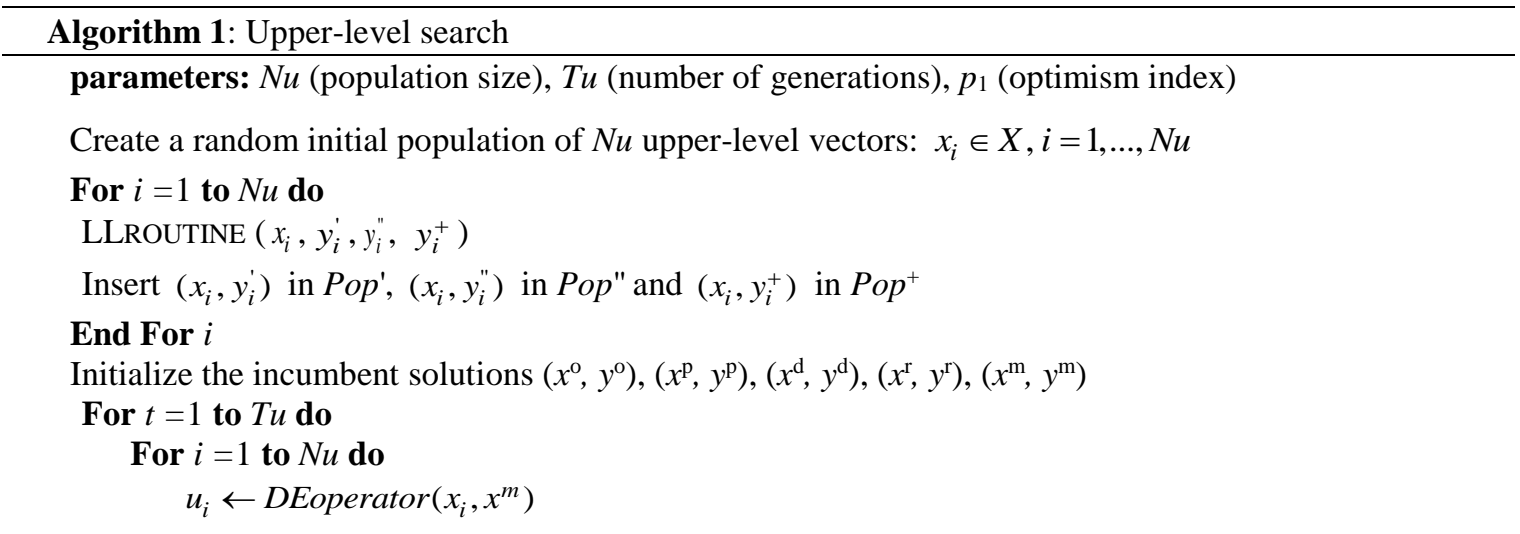


LLROUTINE $\left(u_{i}, w_{i}^{\prime}, w_{i}^{\prime \prime}, w_{i}^{+}\right)$

Update the incumbent solutions with $\left(u_{i}, w_{i}^{\prime}\right),\left(u_{i}, w_{i}^{\prime \prime}\right),\left(u_{i}, w_{i}^{+}\right)$if these improve the current ones.

If $\operatorname{ACCEPT}\left(u_{i}\right)$ then

$x_{i} \leftarrow u_{i}$ and replace the $i$-th solutions of Pop',$P o p^{\prime \prime}$ and Pop $^{+}$with $\left(u_{i}, w_{i}^{\prime}\right),\left(u_{i}, w_{i}^{\prime \prime}\right)$ and $\left(u_{i}, w_{i}^{+}\right)$, respectively.

End For $i$

End For $t$

Output: $\left(x^{\mathrm{o}}, y^{\mathrm{o}}\right),\left(x^{\mathrm{d}}, y^{\mathrm{d}}\right),\left(x^{\mathrm{p}}, y^{\mathrm{p}}\right),\left(x^{\mathrm{r}}, y^{\mathrm{r}}\right),\left(x^{\mathrm{m}}, y^{\mathrm{m}}\right)$

The LLRoutine $\left(x, y^{\prime}, y^{\prime \prime}, y^{+}\right)$aims at computing, for a given $x$, two extreme efficient solutions to the lower-level problem, one belonging to the optimistic frontier, $\left(x, y^{\prime}\right) \in O f$, and another belonging to the pessimistic frontier, $\left(x, y^{\prime \prime}\right) \in P f$, plus the follower's efficient solution $\left(x, y^{+}\right)$in the moderate frontier. This routine employs two phases: phase 1 attempts to converge to a population Popy of efficient solutions to the lower-level problem polarized to the extreme values of $F$, the minimum and the maximum; the phase 2 is oriented to more central follower's efficient solutions, in order to properly approximate the moderate solution.

Let $T l$ be the number of lower-level generations in each phase. The number of individuals $y$ in a lowerlevel search is $\mathrm{Nl}$ (an even number).

In the phase 1, the first $N l / 2$ individuals of Popy are oriented to $y^{\prime}$ while the remaining $N l / 2$ individuals are oriented to $y^{\prime \prime}$. Therefore, for each $y_{i} \in$ Popy, the DEoperator randomly selects $r_{1} \neq r_{2}$, with $r_{1}, r_{2}$ $\in\{1, \ldots, N l / 2\}$ for $i \leq N l / 2$ and $r_{1}, r_{2} \in\{N l / 2+1, \ldots, N l\}$ for $i>N l / 2 ; y_{\text {best }}$ is $y^{\prime}$ for the first half part of the population and $y^{\prime \prime}$ for the second part. In the phase 2 , there is no division of the population and $y_{\text {best }}=y^{+}$. A trial vector $v_{i}$ is created by the DEoperator for each $y_{i}$

Whenever a lower-level $y_{i}$ is randomly generated or a trial vector $v_{i}$ is computed, box constraints (if they exist) are ensured by pulling the variables out of bounds into the closest bounds. If more general constraints $g\left(x, y_{i}\right) \leq 0$ are violated, then another $y_{i}$ is drawn in the initial population for at most three trials; if all these trials are infeasible, the solution with the smallest overall violation of constraints is selected. During the evolution process, solutions that do not satisfy general constraints are penalized in the acceptance criterion.

The acceptance criterion (ACCEPT_LL function in Algorithm 2) determines whether the new individual $v_{i}$ is accepted or not to replace $y_{i}$ in the next population. It firstly observes whether the solutions $\left(x, v_{i}\right)$ and $\left(x, y_{i}\right)$ satisfy the lower-level constraints $g(x, y) \leq 0$, privileging the feasible solution if one of them is infeasible. If both solutions satisfy the constraints, then it is checked whether they are nondominated with respect to the current set of efficient solutions (Eff). If one of the solutions $v_{i}$ or $y_{i}$ is nondominated (i.e., it belongs to Eff) and the other is dominated, the nondominated solution is selected. If both solutions have the same status, the selection is based upon the upper-level objective function value: in Phase 1, for $i \leq N l / 2$ (subpopulation oriented to the $O f$ ) the individual with highest $F$ is selected; for $i>N l / 2$ (sub-population oriented to $P f$ ) the individual with lowest $F$ is selected; in Phase $2, F^{*}=p_{1} F\left(x, y^{\prime}\right)+\left(1-p_{1}\right) F\left(x, y^{\prime \prime}\right)$ is used as the reference value and the solution $\left(x, v_{i}\right)$ or $\left(x, y_{i}\right)$ with $F$ closest to $F^{*}, F \leq F^{*}$, is chosen.

Algorithm 2: LLROUTINE $\left(x \downarrow, y^{\prime} \uparrow, y^{\prime \prime} \uparrow, y^{+} \uparrow\right)$

\section{Phase 1:}

Step 1 - Create a random initial population: Popy $=\left\{y_{i} \in Y, i=1, \ldots, N l\right\}$

Step 2 - Define Eff with the solutions in Popy that satisfy $g\left(x, y_{i}\right) \leq 0$ and are mutually nondominated.

Step 3 - Initialize $y^{\prime}$ and $y^{\prime \prime}$ :

$$
y^{\prime} \leftarrow \underset{y}{\arg \max }\{F(x, y): y \in E f f\} ; y^{\prime \prime} \leftarrow \underset{y}{\arg \min }\{F(x, y): y \in E f f\}
$$

Step 4 -

For $t=1$ to $T l$ do

For $i=1$ to $N l$ do

If $i \leq N l / 2$ then 


\section{End For $i$}

Else $\quad v_{i} \leftarrow$ DEoperator $\left(y_{i}, y^{\prime \prime}\right)$

Insert in Eff the mutually nondominated $\left(x, v_{i}\right), i=1, \ldots, N l$ that satisfy $g\left(x, v_{i}\right) \leq 0$ and are not dominated by any member of Eff. Delete solutions that become dominated in Eff.

For $i=1$ to $N l$ do

$$
\begin{gathered}
\text { If }\left(i \leq N l / 2 \text { and ACCEPT_LL }\left(v_{i}, \text { highest } F\right)\right. \text { or } \\
\left(i>N l / 2 \text { and ACCEPT_LL }\left(v_{i}, \text { lowest } F\right)\right. \text { then } \\
\text { Replace } y_{i} \text { in Popy with } v_{i}
\end{gathered}
$$

End For $i$

Update the incumbent solutions $y^{\prime}$ and $y^{\prime \prime}$ as in Step 3

\section{End For $t$}

\section{Phase 2:}

Let $F^{*}=p_{1} F\left(x, y^{\prime}\right)+\left(1-p_{1}\right) F\left(x, y^{\prime \prime}\right)$

Steps 1 and 2 - similar to steps 1 and 2 of phase 1 .

Step 3 - Initialize $y^{+}: y^{+} \leftarrow \arg \max \left\{F(x, y): y \in \operatorname{Eff}, F(x, y) \leq F^{*}\right\}$

Step 4 -

For $t=1$ to $T l$ do

For $i=1$ to $\mathrm{Nl}$ do

End For $i$

$$
v_{i} \leftarrow \operatorname{DEoperator}\left(y_{i}, y^{+}\right)
$$

Insert in Eff the mutually nondominated $\left(x, v_{i}\right), i=1, \ldots, N l$ that satisfy $g\left(x, v_{i}\right) \leq 0$ and are not dominated by any member of Eff. Delete solutions that become dominated in Eff.

For $i=1$ to $\mathrm{Nl}$ do

If ACCEPT_LL $\left(v_{i}, F^{*}\right)$ then

Replace $y_{i}$ in Popy with $v_{i}$

End For $i$

Update the incumbent solution $y^{+}$as in Step 3 of phase 2 .

End For $t$

Output: $y^{\prime}, y^{\prime \prime}, y^{+}$

Some options adopted in this algorithm have been tuned within the algorithm for Phase 1 presented in (Alves and Antunes, 2018a). For instance, in a first version of the algorithm, the set Eff was not used for the acceptance criterion, which only compared the two candidate solutions, $v_{i}$ and $y_{i}$, checking whether one dominated the other or not. However, the algorithm revealed a very poor convergence of the population to efficient solutions, which was overcome with the current strategy.

Eff is sorted by decreasing order of $F$ and a predefined maximum size $L$ is imposed in order to control the growth of $E f f$. In each generation, if $|E f f|>L$, some elements are successively removed until $L$ solutions remain in Eff: in Phase 1 the removed elements are the most central ones (i.e. the most distant from the maximum and the minimum of $F$ ), while in Phase 2 the opposite is done; the first removed elements are the ones in the top of Eff with $F>F^{*}$ and then, if necessary, elements in the bottom are removed.

Note that the lower-level search scheme employed in this algorithm combines the search for the optimistic and pessimistic frontiers in the same phase because: (a) when the optimistic solution is updated with a solution in the optimistic frontier, the corresponding solution (with the same $x$ ) in the pessimistic frontier should also be available to update the deceiving solution; equivalently for updating the pessimistic and rewarding solutions; (b) solutions that are interesting for one frontier may emerge in the sub-population oriented to the other frontier. The lower-level search for the moderate frontier is performed separately in a second phase because $y^{\prime}$ and $y^{\prime \prime}$ (obtained in the first phase) are needed to define the expected value of $F$, according to the EVP defined above. Moreover, finishing first the search for these solutions may expectedly lead to better approximate solutions in the moderate frontier.

It is worthwhile to mention that the DE algorithm presented herein also computes the solutions on the optimistic frontier and on the pessimistic frontier associated with the moderate solution, i.e., the rewarding and deceiving outcomes of the moderate approach for a given $p_{1}$. For the sake of readability of the algorithm, 
these solutions, which are initialized and updated together with the moderate solution, have been omitted in the formal presentation of the algorithm.

\subsection{Illustrative results}

The DE algorithm was implemented in Delphi language (Embarcadero®). In order to illustrate results of the DE algorithm and possible pitfalls associated with their interpretation, we will use the problem of Example 2 for which exact solutions are known.

We have used the following parameter values: $N u=50 ; T u=50 ; N_{l}=100 ; T_{l}=100 ; L=100$. We have considered 3 values for the optimism index: $p_{1}=0.25 ; p_{1}=0.5 ; p_{1}=0.75$. Thirty independent runs of the algorithm were performed for each value of $p_{1}$, each one aiming at approximating the four extreme solutions and the moderate solution corresponding to that value of $p_{1}$. Since the same seed value was used for the generation of random numbers, the results for the four extreme solutions are the same for all values of $p_{1}$.

Table 1 presents the average and the standard deviation of $F$ obtained for the four extreme solutions and the moderate solutions over the 30 runs. Table 2 shows the values of $F, f_{1}, f_{2}$ and all variables in the best (minimum $F$ ) optimistic, pessimistic and moderate solutions and in the corresponding deceiving/rewarding solutions. In order to facilitate the analysis and assess the quality of the results obtained, we also include the exact values (obtained analytically), which are in bold face near each approximate value.

Table 1. Average and standard deviation of $F$ in Example 2, obtained in 30 independent runs for each $p_{1}$ value.

\begin{tabular}{|c|c|c|c|c|}
\hline \multicolumn{2}{|c|}{ Example 2} & Average $F$ & Stand. Dev. F & Exact \\
\hline & Sol.o & 0.49832 & 0.00051 & 0.5 \\
\hline & Sol.d & 1.24924 & 0.01388 & 1.25 \\
\hline & Sol.p & 0.99445 & 0.00098 & 1 \\
\hline & Sol.r & 0.99047 & 0.00470 & 1 \\
\hline$p_{1}=0.25$ & Sol.m & 0.94432 & 0.00050 & 0.95 \\
\hline$p_{1}=0.5$ & Sol.m & 0.82923 & 0.00067 & $\mathbf{0 . 8 3 3 3 3}$ \\
\hline$p_{1}=0.75$ & Sol.m & 0.67586 & 0.00066 & 0.67857 \\
\hline
\end{tabular}

Table 2. Best optimistic, pessimistic (and corresponding deceiving, rewarding) and moderate solutions.

\begin{tabular}{|c|c|c|c|c|c|c|c|c|c|c|c|c|c|}
\hline \multicolumn{2}{|c|}{ Example 2} & $F$ & & $f_{1}$ & & $f_{2}$ & & $x$ & & $y_{1}$ & & $y_{2}$ & \\
\hline & Sol.o & 0.49729 & 0.5 & 0.26525 & 0.25 & 0.00001 & 0 & 0.51195 & 0.5 & 0.51502 & 0.5 & -0.0010 & 0 \\
\hline & Sol.d & 1.25887 & 1.25 & 0 & 0 & 0.26044 & 0.25 & 0.51195 & 0.5 & 0.00161 & 0 & 0.00016 & 0 \\
\hline & Sol.p & 0.99375 & 1 & 0.00001 & 0 & 0 & 0 & 0.00407 & 0 & 0.00314 & 0 & 0.00019 & 0 \\
\hline & Sol.r & 0.99375 & 1 & 0.00001 & 0 & 0 & 0 & 0.00407 & $\mathbf{0}$ & 0.00314 & $\mathbf{0}$ & 0.00019 & 0 \\
\hline$p_{1}=0.25$ & Sol.m & 0.94301 & 0.95 & 0.0028 & 0.00212 & 0.02612 & 0.0237 & 0.21453 & 0.2 & 0.05291 & 0.04606 & 0.00144 & 0 \\
\hline$p_{1}=0.5$ & Sol.m & 0.82754 & 0.83333 & 0.0224 & 0.02255 & 0.03012 & 0.03355 & 0.32321 & 0.33333 & 0.14966 & 0.15016 & 0.00014 & 0 \\
\hline$p_{1}=0.75$ & Sol.m & 0.67458 & 0.67857 & 0.08443 & 0.08792 & 0.0152 & 0.01744 & 0.41387 & 0.42857 & 0.29057 & 0.29651 & 0.00013 & 0 \\
\hline
\end{tabular}

Figure 15 displays a screenshot of the algorithm being run with Example 2, showing the populations and the current incumbent solutions (extreme solutions and moderate solution for $p_{1}=0.5$ ) after 10 iterations, i.e., 10 upper-level generations.

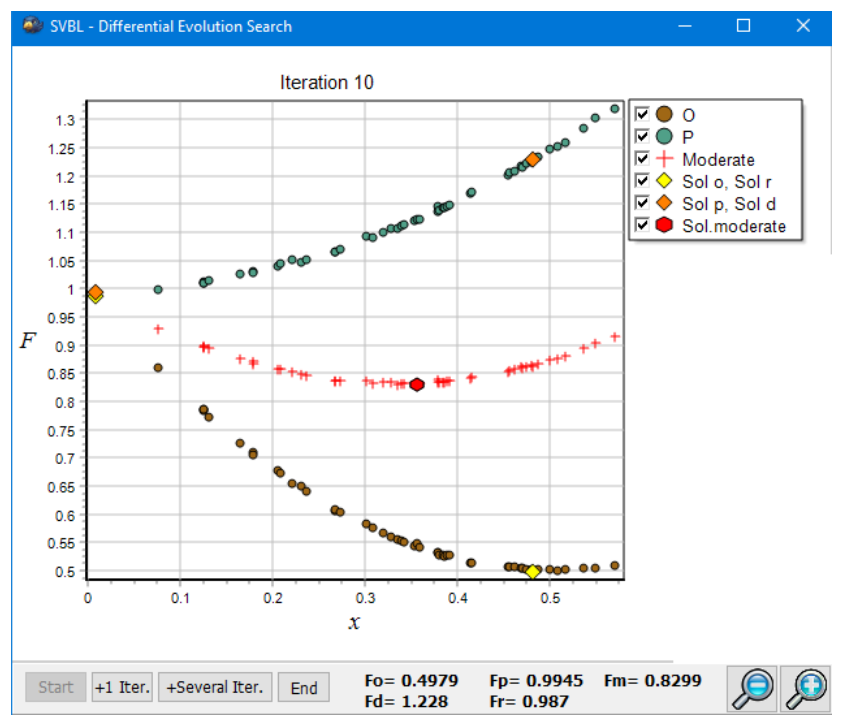


Figure 15- Screen of the algorithm being run with Example 2 (for $p_{1}=0.5$ ).

When a heuristic algorithm is applied to an optimization problem, the intention is to obtain an optimal or near-optimal solution; so, the solution with minimum objective function value (assuming minimization, as in this problem) is chosen. This is what we have done to select the solutions presented in Table 2. However, there are additional issues in assessing solutions in SVBP and MOBP that can lead to misleading interpretations. These pitfalls are very difficult to avoid in general problems for which the exact solutions are not known. Nevertheless, we wish to draw attention to some of them using this example.

Consider, for instance, the best solution (presented in Table 2) for the optimistic solution. It has $F=$ 0.49729 , which is better than the real value, $F=0.5$. The optimistic solution is the best solution to the leader in the whole induced region, so the reason to obtain a better $F$ value is that the solution is infeasible because it is not efficient to the lower level problem. The upper-level solution in Sol.o is $x=0.51195$; we can realize that, for this $x$, the lower-level solution that is efficient and the best for the leader is $\left(y_{1}, y_{2}\right)=(0.51195,0)$, with $\left(f_{1}, f_{2}\right)=(0.26209,0)$ leading to $F=0.5003$. Note that $\left(f_{1}, f_{2}\right)=(0.26209,0)$ dominates $\left(f_{1}, f_{2}\right)=(0.26525$, $0.0001)$ in Sol.o. $F=0.5003$ is worse than the true optimal value $(0.5003>0.5)$ because the optimal optimistic solution is obtained for a different $x$.

Actually, only efficient solutions to the lower-level problem are feasible to the SVBP, but this is very difficult to ensure using approximate methods, leading the algorithm to yield apparently better results with invalid solutions. Therefore, during the evolution process, an exact optimal solution (in any frontier) can be disregarded because it is worse in $F$ than other solutions that are not efficient to the lower-level problem (although they may be very close to the efficient solution set).

But, even when the lower-level solutions are efficient, other difficulties arise in evaluating solutions in frontiers other than the optimistic one. Consider, for instance, solutions in the pessimistic frontier: approximate solutions may have an $F$ value better than the true optimal values because they are not the worst for the leader for that specific value of $x$, i.e., they are not really in the pessimistic frontier. This drawback may lead to retain false pessimistic and deceiving solutions, which may also occur in the computation of solutions in other frontiers.

Moreover, solutions closer to the optimal ones may be obtained in other runs of the algorithm. However, since they have a worse $F$ value, they are not chosen as the best solutions. Consider, for instance, the moderate solution for $p_{1}=0.5$, for which the exact $F$ value is 0.83333 and $\left(x, y_{1}, y_{2}\right)=(0.33333,0.15016,0)$. The solution selected as the best one (presented in Table 2$)$ is the one with best (lower) $F:\left(x, y_{1}, y_{2}\right)=(0.32321,0.14966$, $0.00014), F=0.82754$. However, we could observe that there are solutions provided by other runs, e.g. $\left(x, y_{1}\right.$, $\left.y_{2}\right)=(0.33537,0.152865,0.00035), F=0.83011$, which could better approximate the moderate solution, although they would never be selected.

Consequently, approximate algorithms, e.g. stochastic optimizers as DE, may lead to apparently better solutions to SVBP/MOBP than the exact optimal/Pareto optimal ones. However, those solutions, even being good approximations to optimal/Pareto optimal solutions, may not be efficient to the lower-level problem, therefore leading to overestimated results. I.e., a better Pareto front does not imply a better performance of the algorithm if the lower-level solutions are not truly efficient for each instantiation of the upper-level variables. The computation and interpretation of results in this type of problems should take thoroughly into account the pitfalls herein illustrated to avoid erroneous conclusions. This issue has been completely disregarded in the literature for the comparison of algorithms; the authors often claim that a new MOBP algorithm outperforms other algorithms based only on the comparison of the respective upper-level Pareto fronts.

\section{$7 \quad$ Conclusions and further research}

A relevant challenge in bilevel problems with multiple objective functions at both levels or only at the lower-level (MOBP/SVBP) is to guarantee the feasibility of solutions, because solutions that are not efficient to the lower-level problem are infeasible to the bilevel problem. In addition, since, in general, the leader does not possess information on the follower's preferences within his efficient region, the effectiveness of the decision support process requires that solutions deriving from more optimistic or more pessimistic leader's attitudes and more favorable or more adverse follower's reactions should be explored.

For this purpose, this paper proposes solution concepts in SVBP and MOBP, which explore the interaction between the leader's and the follower's decisions to define: - in SVBP, the optimistic and the pessimistic frontiers, where the extreme optimistic/deceiving and pessimistic/rewarding solutions are located, as well as moderate solutions according to the risk the leader is willing to take; - in MOBP, the Optimistic and the 
Pessimistic Pareto Fronts. These novel concepts are illustrated through graphical examples to cast light on the insights these solutions can offer to support decision processes based on SVBP and MOBP models.

We have paid particular attention to pessimistic approaches since most algorithms developed thus far for SVBP/MOBP are mainly dedicated to the computation of the optimistic solution/Pareto front (which subsumes the unlikely assumption that the follower is indifferent to all efficient solutions obtained for a given decision of the leader). The graphical examples of linear and nonlinear problems enabled to ascertain critical issues, such as that the Pessimistic Pareto Front may include part of the Optimistic Pareto Front. The definitions of Optimistic and the Pessimistic Pareto Fronts herein proposed lay the foundations for the development of algorithms to compute them so that the leader can be offered information about the risk vs. opportunity associated with his decisions.

Population-based meta-heuristics have been increasingly used to deal with SVBP/MOBP. It should be noted that, being approximate algorithms, these approaches may lead to seemingly better solutions. However, these solutions may not be actually efficient to the lower-level problem (even being good approximations) and therefore be taken by the algorithms in detriment of the true efficient solutions thus leading to misleading results. I.e., a better front does not imply a better performance of the algorithm if the efficiency of lowerlevel solutions is not ensured. An algorithm based on differential evolution for the SVBP has been presented, which aims to approximate the extreme and moderate solutions. The discussion of the results of this algorithm enabled to highlight those pitfalls.

The judicious combination of meta-heuristics for the upper-level search with exact solvers for the lowerlevel problem can contribute to mitigate that drawback, in cases where this combination is possible (e.g., when the lower-level problem is a mixed-integer linear programming problem after the instantiation of the upper-level variables).

Further work will include the development of algorithms to approximate the Optimistic and the Pessimistic Pareto Fronts of the MOBP.

Acknowledgment. This work was supported by projects UID/MULTI/00308/2013, ESGRIDS (POCI-010145-FEDER-016434), MAnAGER (POCI-01-0145-FEDER-028040), SUSpENsE (CENTRO-01-0145FEDER-000006) and RTCARE (POCI-01-0145-FEDER-028030).

\section{Compliance with Ethical Standards}

Conflict of Interest: The authors declare that they have no conflict of interest.

Ethical approval: This article does not contain any studies with human participants or animals performed by any of the authors.

\section{References}

Abo-Sinna, M. A. and Baky, I. A. (2007) 'Interactive Balance Space Approach for Solving Multi-level Multi-objective Programming Problems', Information Sciences, 177(16), pp. 3397-3410. doi: 10.1016/j.ins.2007.02.005.

Alves, M. J. and Antunes, C. H. (2018a) 'A differential evolution algorithm to semivectorial bilevel problems', in Nicosia, G. et al. (eds) Machine Learning, Optimization, and Big Data. MOD 2017. Lecture Notes in Computer Science. Springer, Cham, pp. 172-185. doi: 10.1007/978-3-319-72926-8_15.

Alves, M. J. and Antunes, C. H. (2018b) 'A semivectorial bilevel programming approach to optimize electricity dynamic time-of-use retail pricing', Computers and Operations Research, 92, pp. 130-144. doi: 10.1016/j.cor.2017.12.014

Alves, M. J., Antunes, C. H. and Carrasqueira, P. (2015) 'A PSO Approach to Semivectorial Bilevel Programming: Pessimistic, Optimistic and Deceiving Solutions', in Proceedings of the Genetic and Evolutionary Computation Conference (GECCO 2015), pp. 599-606. doi: 10.1145/2739480.2754644.

Alves, M. J., Antunes, C. H. and Costa, J. P. (2019) 'Multiobjective Bilevel Programming: Concepts and Perspectives of Development', in Doumpos, M. et al. (eds) New Perspectives in Multiple Criteria Decision Making: Innovative Applications and Case Studies. Cham: Springer International Publishing, pp. 267-293. doi: 10.1007/978-3-030-11482-4_10.

Ankhili, Z. and Mansouri, A. (2009) 'An exact penalty on bilevel programs with linear vector optimization lower level', European Journal of Operational Research, 197(1), pp. 36-41. doi: 10.1016/j.ejor.2008.06.026.

Bard, J. (1998) 'Nonconvex Optimization and Its Applications'. Dordrecht: Kluwer Academic. doi: 10.1007/978-1-4757-2836-1.

Bonnel, H. (2006) 'Optimality conditions for the semivectorial bilevel optimization problem', Pacific 
Journal of Optimization, 2(3), pp. 447-468.

Calvete, H. and Galé, C. (2011) 'On linear bilevel problems with multiple objectives at the lower level', Omega, 39(1), pp. 33-40. doi: 10.1016/j.omega.2010.02.002.

Carrasqueira, P., Alves, M. J. and Antunes, C. H. (2015) 'A Bi-level Multiobjective PSO Algorithm', in Gaspar-Cunha, A., Antunes, C. H., and Coello Coello, C. (eds) Evolutionary Multi-Criterion Optimization (EMO 2015), Lecture Notes in Computer Science 9018. Springer International Publishing, pp. 263-276. doi: 10.1007/978-3-319-15934-8_18.

Colson, B., Marcotte, P. and Savard, G. (2005) 'Bilevel programming: A survey', 4OR, 3(2), pp. 87-107. doi: 10.1007/s10288-005-0071-0.

Colson, B., Marcotte, P. and Savard, G. (2007) 'An overview of bilevel optimization', Annals of Operations Research, 153(1), pp. 235-256. doi: 10.1007/s10479-007-0176-2.

Dassanayaka, S. M. (2010) 'Methods Of Variational Analysis In Pessimistic Bilevel Programming', Wayne State University Dissertations, 126.

Deb, K. and Sinha, A. (2009) 'Solving bilevel multi-objective optimization problems using evolutionary algorithms', in Proc. of EMO 2009, Lecture Notes in Computer Science 5467. Springer, pp. 110-124.

Deb, K. and Sinha, A. (2010) 'An efficient and accurate solution methodology for bilevel multi-objective programming problems using a hybrid evolutionary-local-search algorithm', Evolutionary computation, 18(3), pp. 403-449.

Dempe, S. (2002) Foundations of bilevel programming. Springer US. doi: 10.1007/b101970.

Eichfelder, G. (2010) 'Multiobjective bilevel optimization', Mathematical Programming, 123(2), pp. 419449. doi: 10.1007/s10107-008-0259-0.

Gupta, A. and Ong, Y. (2015) 'An evolutionary algorithm with adaptive scalarization for multiobjective bilevel programs', in 2015 IEEE Congress on Evolutionary Computation (CEC), Sendai, 2015, pp. 16361642.

Labbé, M. and Violin, A. (2013) 'Bilevel programming and price setting problems', 4OR, 11(1), pp. 130. doi: 10.1007/s10288-012-0213-0.

Liu, B. et al. (2014) 'Optimality conditions for pessimistic semivectorial bilevel programming problems', Journal of Inequalities and Applications, 2014, p. 41. doi: 10.1186/1029-242X-2014-41.

Lucchetti, R., Mignanego, F. and Pieri, G. (1987) 'Existence theorems of equilibrium points in stackelberg', Optimization, 18(6), pp. 857-866. doi: 10.1080/02331938708843300.

Lv, Y. and Chen, J. (2016) 'A Discretization Iteration Approach for Solving a Class of Semivectorial Bilevel Programming Problem', Journal of Nonlinear Sciences \& Applications (JNSA), 9(5), pp. 2888-2899. doi: 10.22436/jnsa.009.05.83.

Lv, Y. and Wan, Z. (2014) 'A solution method for the optimistic linear semivectorial bilevel optimization problem', Journal of Inequalities and Applications, 2014(1), p. 164. doi: 10.1186/1029-242X-2014-164.

Pieume, C. et al. (2011) 'Solving Bilevel Linear Multiobjective Programming Problems', American Journal of Operations Research, 1, pp. 214-219. doi: 10.4236/ajor.2011.14024.

Price, K., Storn, R. M. and Lampinen, J. A. (2006) Differential evolution: a practical approach to global optimization. Springer-Verlag Berlin Heidelberg. doi: 10.1007/3-540-31306-0.

Ren, A. and Wang, Y. (2016) 'A novel penalty function method for semivectorial bilevel programming problem', Applied Mathematical Modelling, 40(1), pp. 135-149. doi: 10.1016/j.apm.2015.04.041.

Ruuska, S. and Miettinen, K. (2012) 'Constructing evolutionary algorithms for bilevel multiobjective optimization', in 2012 IEEE Congress on Evolutionary Computation (CEC). IEEE, pp. 1-7. doi: 10.1109/CEC.2012.6256156.

Shi, X. and Xia, H. (1997) 'Interactive bilevel multi-objective decision making', Journal of the Operational Research Society, 48(9), pp. 943-949.

Shi, X. and Xia, H. (2001) 'Model and interactive algorithm of bi-level multi-objective decision-making with multiple interconnected decision makers', Journal of Multi-Criteria Decision Analysis, 10, pp. 27-34.

Sinha, A. et al. (2016) 'Solving Bilevel Multicriterion Optimization Problems With Lower Level Decision Uncertainty', IEEE Transactions on Evolutionary Computation, 20(2), pp. 199-217. doi: 10.1109/TEVC.2015.2443057.

Sinha, A., Malo, P. and Deb, K. (2017) 'Evolutionary Bilevel Optimization: An Introduction and Recent Advances', in Bechikh, S., Datta, R., and Gupta, A. (eds) Recent Advances in Evolutionary Multi-objective Optimization. Springer, Cham, pp. 71-103.

Sinha, A., Malo, P. and Deb, K. (2018) 'A Review on Bilevel Optimization: From Classical to Evolutionary Approaches and Applications', IEEE Transactions on Evolutionary Computation, 22(2), pp. 276-295. doi: 10.1109/TEVC.2017.2712906.

Vicente, L. and Calamai, P. (1994) 'Bilevel and multilevel programming: a bibliography review', Journal 
of Global Optimization, 5(3), pp. 291-306. doi: 10.1007/BF01096458.

Zhang, T. et al. (2013) 'Solving high dimensional bilevel multiobjective programming problem using a hybrid particle swarm optimization algorithm with crossover operator', Knowledge-Based Systems, 53, pp. 13-19. doi: 10.1016/j.knosys.2013.07.015.

Zheng, Y., Chen, J. and Cao, X. (2014) 'A Global Solution Method for Semivectorial Bilevel Programming Problem', Filomat, 28(8), pp. 1619-1627.

Zheng, Y. and Wan, Z. (2011) 'A solution method for semivectorial bilevel programming problem via penalty method', Journal of Applied Mathematics and Computing, 37(1-2), pp. 207-219. doi: 10.1007/s12190-010-0430-7. 